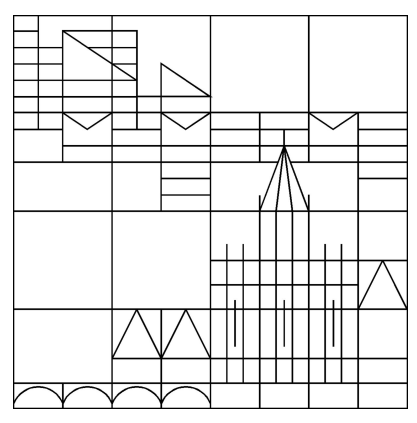

\title{
On the stability of damped Timoshenko systems - Cattaneo versus Fourier law
}

\author{
Hugo D. Fernández Sare \\ Reinhard Racke
}

Konstanzer Schriften in Mathematik und Informatik

Nr. 227, März 2007

ISSN 1430-3558

Konstanzer Online-Publikations-System (KOPS)

URL: http://www.ub.uni-konstanz.de/kops/volltexte/2007/2408/

URN: http://nbn-resolving.de/urn:nbn:de:bsz:352-opus-24085

(C) Fachbereich Mathematik und Statistik
(C) Fachbereich Informatik und Informationswissenschaft
Universität Konstanz
Fach D 188, 78457 Konstanz, Germany
E-Mail: preprints@informatik.uni-konstanz.de
WWW: http://www.informatik.uni-konstanz.de/Schriften/ 


\title{
On the stability of damped Timoshenko systems - Cattaneo versus Fourier law*
}

\author{
Hugo D. Fernández Sare and Reinhard Racke
}

\begin{abstract}
We consider vibrating systems of hyperbolic Timoshenko type that are coupled to a heat equation modeling an expectedly dissipative effect through heat conduction. While proving exponential stability under the Fourier law of heat conduction, it turns out that the coupling via the Cattaneo law does not yield an exponentially stable system. This seems to be the first example that a removal of the paradox of infinite propagation speed inherent in Fourier's law by changing to the Cattaneo law distroys the exponential stability property. Actually, for systems with history, the Fourier law keeps the exponential stability known for the pure Timoshenko system without heat conduction, but introducing the Cattaneo coupling even destroys this property.
\end{abstract}

\section{Introduction}

The classical model for the propagation of heat turns into the well-known equations for the temperature $\theta$ (difference to a fixed constant reference temperature) and the heat flux vector $\mathbf{q}$,

$$
\theta_{t}+\beta \operatorname{div} \mathbf{q}=0
$$

and

$$
\mathbf{q}+\kappa \nabla \theta=0
$$

with positive constants $\beta, \kappa$. Relation (1.2) represents the assumed Fourier's law of heat conduction and, plugged into (1.1), yields the parabolic heat equation

$$
\theta_{t}-\beta \kappa \Delta \theta=0 \text {. }
$$

Adding initial conditions and, for example, Dirichlet boundary conditions for $\theta$ we obtain the exponential decay of solutions to (1.3), the associated one parameter semigroup is exponentially stable.

The model using Fourier's law inhibits the physical paradox of infinite propagation speed of signals. For some applications like working with very short laser pulses in laser cleaning of computer chips, see the references in [14], it is worth while thinking of another model removing this paradox, but still keeping the essentials of a heat conduction process. One such model for a survey compare Chandrasekharaiah [2], for general Cattaneo models cp. Öncü and Moodie [13] — is given by the simplest Cattaneo law replacing Fourier's law (1.2),

$$
\tau \mathbf{q}_{t}+\mathbf{q}+\kappa \nabla \theta=0
$$

\footnotetext{
${ }^{0}$ AMS subject classification: 35 B 40, 74 H 40.

${ }^{0}$ Keywords: exponential stability, second sound, heat conduction models.

*This work was supported by the DFG-project "Hyperbolic Thermoelasticity" (RA 504/3-2).
} 
now regarding the heat flux vector as another function to be determined through the differential equation and initial and, in case, boundary conditions. The positive parameter $\tau$ is the relaxation time describing the time lag in the response of the heat flux to a gradient in the temperature. Combining (1.1) and (1.4) we obtain the hyperbolic, damped wave equation

$$
\tau \theta_{t t}+\theta_{t}-\beta \kappa \Delta \theta=0 .
$$

Again, we obtain the well-known exponential stability. That is, both models, Fourier and Cattaneo, exhibit the same qualitative behavior, they both lead to exponentially stable systems for pure heat conduction.

There are many coupled systems describing both the elastic behavior of a system as well as simultaneously the heat conduction within the system. Such thermoelastic systems have been treated by many authors, for a survey on classical thermoelasticity - classical here also indicating that the Fourier law for heat conduction is used — see e.g. [7]. It has been shown that spacially one-dimensional systems are, under appropriate boundary conditions or normalizations, exponentially stable in bounded reference configurations. In three space dimensions the same holds for radially symmetric situations.

This has been extended to models where the Fourier law is replaced by the Cattaneo law in $[14,15,10,5]$. Moreover, it has been shown in the one-dimensional frame work, that, for real materials, the decay rates (type of the associated semigroup) of solutions to the both models are very close to each other, see [6], and that, again for real materials in the model of pulsed laser heating, differences for the displacement or the displacement gradient are of order $10^{-5} \mathrm{~m}$ and $10^{-10} \mathrm{~m}$, respectively, cp. [5].

These observations nourish the expectation that always both models lead to exponential stability (or both do not). We shall demonstrate for Timoshenko type systems that Fourier's law might predict exponential stability, while Cattaneo's law does not. This observation seems to be new and, maybe, unexpected. It turns out that for Timoshenko systems with history which are known to decay exponentially due to the history the introduction of a heat conduction via Fourier keeps this exponential decay property while the Cattaneo model even destroys this property.

The first system we consider is the following coupling of two wave equations of Timoshenko type with heat conduction

$$
\begin{aligned}
\rho_{1} \varphi_{t t}-k\left(\varphi_{x}+\psi\right)_{x} & =0 & & \text { in }(0, \infty) \times(0, L) \\
\rho_{2} \psi_{t t}-b \psi_{x x}+k\left(\varphi_{x}+\psi\right)+\delta \theta_{x} & =0 & & \text { in }(0, \infty) \times(0, L) \\
\rho_{3} \theta_{t}+q_{x}+\delta \psi_{t x} & =0 & & \text { in }(0, \infty) \times(0, L) \\
\tau q_{t}+\beta q+\theta_{x} & =0 & & \text { in }(0, \infty) \times(0, L)
\end{aligned}
$$

with positive constants $\rho_{1}, k, \rho_{2}, b, \delta, \rho_{3}, \beta$.

The case $\tau=0$ represents Fourier's law, and $\tau>0$ Cattaneo's law. The functions $\varphi, \psi, \theta$ and $q$ depend on $(t, x) \in[0, \infty) \times[0, L]$ and model the transverse displacement of a beam with reference configuration $(0, L) \subset \mathbb{R}$, the rotation angle of a filament, the temperature difference and the heat flux, respectively, cp. [8].

Additionally we have initial conditions

$$
\varphi(0, \cdot)=\varphi_{0}, \varphi_{t}(0, \cdot)=\varphi_{1}, \psi(0, \cdot)=\psi_{0}, \psi_{t}(0, \cdot)=\psi_{1},
$$




$$
\theta(0, \cdot)=\theta_{0}, q(0, \cdot)=q_{0} \quad \text { in }(0, L)
$$

(the last one for $q$ only if $\tau>0$ ), and boundary conditions

$$
\varphi(\cdot, 0)=\varphi(\cdot, L)=\psi_{x}(\cdot, 0)=\psi_{x}(\cdot, L)=\theta(\cdot, 0)=\theta(\cdot, L)=0 \quad \text { in }(0, \infty)
$$

It was shown in [11] that for $\tau=0$, i.e. assuming Fourier's law, the system is exponentially stable if and only if

$$
\frac{\rho_{1}}{k}=\frac{\rho_{2}}{b}
$$

holds. If the term $\delta \theta_{x}$ in (1.7) is replaced by a control function $\bar{b}(x) \psi_{t}, \bar{b}>0$, then Soufyane [16] proved the exponential stability of the linearized system if and only if (1.12) holds, that is, if and only if the wave speeds associated to (1.6), (1.7), respectively, are equal.

A weaker type of dissipation, also being presented only in the equation (1.7) for $\psi$, was considered in [1] replacing $\delta \theta_{x}$ by a memory term $\int_{0}^{t} g(t-s) \psi_{x x}(s, x) d s$. For exponential type kernels $g$ the exponential stability follows again if and only if (1.12) holds.

Here we consider a dissipation through a coupling to a heat equation. The coupling is direct only for the rotation angle $\psi$ in (1.7) while the coupling to $\varphi$ is only given indirectly in (1.6). For $\delta=0$ the equations (1.6), (1.7) build an energy conserving purely hyperbolic system. For $\delta \neq 0$ and $\tau=0$, our system (1.6)-(1.9) is of hyperbolic-parabolic type, while for $\tau>0$ it is damped, purely hyperbolic.

We shall prove that, under the same condition (1.12), the system is no longer exponentially stable under Cattaneo's law where $\tau>0$. Thus the behavior under the Fourier law is essentially different from the behavior under Cattaneo's law, which, for the question of stability might not have been expected.

Then we can even add another kind of dissipation given through a history term. We look at the extended system in $(0, \infty) \times(0, L)$,

$$
\begin{aligned}
\rho_{1} \varphi_{t t}-k\left(\varphi_{x}+\psi\right)_{x} & =0 \\
\rho_{2} \psi_{t t}-b \psi_{x x}+\int_{0}^{\infty} g(s) \psi_{x x}(t-s, \cdot) d s+k\left(\varphi_{x}+\psi\right)+\delta \theta_{x} & =0 \\
\rho_{3} \theta_{t}+q_{x}+\delta \psi_{t x} & =0 \\
\tau q_{t}+\beta q+\theta_{x} & =0
\end{aligned}
$$

where the integral term in (1.14) represents a history term with an exponentially decaying kernel $g$, cp. [3] for the purely hyperbolic system (1.13), (1.14) without heat conduction, and [1] for finite history without heat conduction. It will be demonstrated that the system is exponentially stable for $\tau=0$ if and only if (1.12) holds, while it is not exponentially stable if $\tau>0$.

Since the system without heat conduction, the pure Timoshenko beam equation ((1.13), $(1.14), \delta=0$ ), is exponentially stable, see [3]), (cp. [1] for finite history), this means that the Fourier model of heat conduction preserves the exponential stability of the model, while the still assumed to have a dissipative effect - Cattaneo model destabilizes in the sense that it is no longer exponentially stable. This discovered phenomenon seems to be unexpected and may have consequences for other hyperbolic heat conduction models.

The paper is organized as follows: In Section 2 we shall look at the Timoshenko system (1.6)(1.9) and prove that it is not exponentially stable for the Cattaneo law $(\tau>0)$ even if $(1.12)$ 
holds. The Timoshenko system with history (1.13)-(1.16) is shown to be not exponentially stable under Cattaneo's law $(\tau>0)$ in Section 3, and to be exponentially stable under the Fourier law in Section 4.

\section{Timoshenko without history — non-exponential stability for Cattaneo's law}

We consider here the initial-boundary value problem (1.6)-(1.11) for the Timoshenko system without history under Cattaneo's law, i.e. $\tau>0$,

$$
\begin{array}{rll}
\rho_{1} \varphi_{t t}-k\left(\varphi_{x}+\psi\right)_{x} & =0 & \text { in }(0, \infty) \times(0, L) \\
\rho_{2} \psi_{t t}-b \psi_{x x}+k\left(\varphi_{x}+\psi\right)+\delta \theta_{x} & =0 & \text { in }(0, \infty) \times(0, L) \\
\rho_{3} \theta_{t}+q_{x}+\delta \psi_{t x} & =0 & \text { in }(0, \infty) \times(0, L) \\
\tau q_{t}+\beta q+\theta_{x} & =0 & \text { in }(0, \infty) \times(0, L) \\
\varphi(0, \cdot)=\varphi_{0}, \varphi_{t}(0, \cdot)=\varphi_{1}, \psi(0, \cdot)=\psi_{0}, \psi_{t}(0, \cdot)=\psi_{1}, \\
\theta(0, \cdot)=\theta_{0}, q(0, \cdot)=q_{0} & \text { in }(0, L) \\
\varphi(\cdot, 0)=\varphi(\cdot, L)=\psi_{x}(\cdot, 0)=\psi_{x}(\cdot, L)=\theta(\cdot, 0)=\theta(\cdot, L)=0 & \text { in }(0, \infty) .
\end{array}
$$

Still assuming the condition (1.12) that was already necessary (and there sufficient) for exponential stability in the Fourier case $(\tau=0)$,

$$
\frac{\rho_{1}}{k}=\frac{\rho_{2}}{b}
$$

we shall demonstrate that exponential stability is no longer given. For this purpose we rewrite the system as evolution equation for $U=\left(\varphi, \varphi_{t}, \psi, \psi_{t}, \theta, q\right)^{\prime} \equiv\left(u^{1}, u^{2}, u^{3}, u^{4}, u^{5}, u^{6}\right)^{\prime}$. Then $U$ formally satisfies

$$
U_{t}=\mathcal{A}_{1} U, \quad U(0)=U_{0}
$$

where $U_{0}:=\left(\varphi_{0}, \varphi_{1}, \psi_{0}, \psi_{1}, \theta_{0}, q_{0}\right)^{\prime}$, and $\mathcal{A}_{1}$ is the (yet formal) differential operator

$$
\mathcal{A}_{1}:=\left(\begin{array}{cccccc}
0 & I d & 0 & 0 & 0 & 0 \\
\frac{k}{\rho_{1}} \partial_{x}^{2} & 0 & \frac{k}{\rho_{1}} \partial_{x} & 0 & 0 & 0 \\
0 & 0 & 0 & I d & 0 & 0 \\
-\frac{k}{\rho_{2}} \partial_{x} & 0 & \frac{b}{\rho_{2}} \partial_{x}^{2}-\frac{k}{\rho_{2}} I d & 0 & -\frac{\delta}{\rho_{2}} \partial_{x} & 0 \\
0 & 0 & 0 & -\frac{\delta}{\rho_{3}} \partial_{x} & 0 & -\frac{1}{\rho_{3}} \partial_{x} \\
0 & 0 & 0 & 0 & -\frac{1}{\tau} \partial_{x} & -\frac{\beta}{\tau} I d
\end{array}\right) .
$$

Let

$$
\mathcal{H}_{1}:=H_{0}^{1}(0, L) \times L^{2}(0, L) \times H_{*}^{1}(0, L) \times L_{*}^{2}(0, L) \times L^{2}(0, L) \times L^{2}(0, L)
$$


be the Hilbert space with

$$
L_{*}^{2}(0, L):=\left\{v \in L^{2}(0, L) \mid \int_{0}^{L} v(x) d x=0\right\}, \quad H_{*}^{1}(0, L):=\left\{v \in H^{1}(0, L) \mid \int_{0}^{L} v(x) d x=0\right\}
$$

and norm given by

$$
\begin{aligned}
\|U\|_{\mathcal{H}_{1}}^{2} & =\left\|\left(u^{1}, u^{2}, u^{3}, u^{4}, u^{5}, u^{6}\right)\right\|_{\mathcal{H}_{1}}^{2} \\
& =\rho_{1}\left\|u^{2}\right\|_{L^{2}}^{2}+\rho_{2}\left\|u^{4}\right\|_{L^{2}}^{2}+b\left\|u_{x}^{3}\right\|_{L^{2}}^{2}+k\left\|u_{x}^{1}+u^{3}\right\|_{L^{2}}^{2}+\rho_{3}\left\|u^{5}\right\|_{L^{2}}^{2}+\tau\left\|u^{6}\right\|_{L^{2}}^{2} .
\end{aligned}
$$

The domain of the operator $\mathcal{A}_{1}$ is given by

$$
\begin{gathered}
D\left(\mathcal{A}_{1}\right):=\left\{U \in \mathcal{H}_{1} \mid u^{1} \in H^{2}(0, L), u^{2} \in H_{0}^{1}(0, L), u^{3} \in H^{2}(0, L), u_{x}^{3} \in H_{0}^{1}(0, L),\right. \\
\left.u^{4} \in H_{*}^{1}(0, L), u^{5} \in H_{0}^{1}(0, L), u^{6} \in H^{1}(0, L)\right\} .
\end{gathered}
$$

It is not difficult to prove that the operator $\mathcal{A}_{1}$ is the infinitesimal generator of a $C_{0}$ contraction semigroup, cp. Section 3.

We shall use the following well-known result from semigroup theory (see e.g. [9, Theorem 1.3.2]).

Lemma 2.1 A semigroup of contractions $\left\{\mathrm{e}^{t \mathcal{A}}\right\}_{t \geq 0}$ in a Hilbert space with norm $\|\cdot\|$ is exponentially stable if and only if

(i) the resolvent set $\varrho(\mathcal{A})$ of $\mathcal{A}$ contains the imaginary axis

and

$$
\text { (ii) } \limsup _{\lambda \rightarrow \pm \infty}\left\|(i \lambda I d-\mathcal{A})^{-1}\right\|<\infty
$$

hold.

Hence it suffices to show the existence of sequences $\left(\lambda_{n}\right)_{n} \subset \mathbb{R}$ with $\lim _{n \rightarrow \infty}\left|\lambda_{n}\right|=\infty$, and $\left(U_{n}\right)_{n} \subset D\left(\mathcal{A}_{1}\right),\left(F_{n}\right)_{n} \subset \mathcal{H}$, such that $\left(i \lambda_{n} I d-\mathcal{A}_{1}\right) U_{n}=F_{n}$ is bounded and

$$
\lim _{n \rightarrow \infty}\left\|U_{n}\right\|_{\mathcal{H}_{1}}=\infty
$$

As $F_{n} \equiv F$ we choose $F:=(0, \sin (\alpha \lambda x), 0, \cos (\alpha \lambda x), 0,0)^{\prime}$, where

$$
\lambda \equiv \lambda_{n}:=\frac{n \pi}{\alpha L} \quad(n \in \mathbb{N}), \quad \alpha:=\sqrt{\frac{\rho_{1}}{k}} .
$$

The solution $U=\left(v^{1}, v^{2}, v^{3}, v^{4}, v^{5}, v^{6}\right)^{\prime}$ of $\left(i \lambda I d-\mathcal{A}_{1}\right) U=F$ should satisfy

$$
\begin{aligned}
i \lambda v^{1}-v^{2} & =0 \\
i \lambda v^{3}-v^{4} & =0 \\
-\lambda^{2} v^{1}-\frac{k}{\rho_{1}} v_{x x}^{1}-\frac{k}{\rho_{1}} v_{x}^{3} & =f^{2} \\
-\lambda^{2} v^{3}-\frac{b}{\rho_{2}} v_{x x}^{3}+\frac{k}{\rho_{2}} v_{x}^{1}+\frac{k}{\rho_{2}} v^{3}+\frac{\delta}{\rho_{2}} v_{x}^{5} & =f^{4} \\
i \lambda v^{5}+\frac{1}{\rho_{3}} v_{x}^{6}+i \lambda \frac{\delta}{\rho_{3}} v_{x}^{3} & =0 \\
i \lambda v^{6}+\frac{\beta}{\tau} v^{6}+\frac{1}{\tau} v_{x}^{5} & =0 .
\end{aligned}
$$


This can be solved by

$$
\begin{array}{ll}
v^{1}(x)=A \sin (\alpha \lambda x), & v^{3}(x)=B \cos (\alpha \lambda x) \\
v^{5}(x)=C \sin (\alpha \lambda x), & v^{6}(x)=D \cos (\alpha \lambda x)
\end{array}
$$

where $A, B, C, D$ depend on $\lambda$ and will be determined explicitly in the sequel. Note that this choice is just compatible with the boundary conditions. System (2.5) is equivalent to finding $A, B, C, D$ such that

$$
\begin{aligned}
-\lambda^{2} A+\frac{k}{\rho_{1}} \alpha^{2} \lambda^{2} A-\frac{k}{\rho_{1}} \alpha \lambda B & =1 \\
-\lambda^{2} B+\frac{b}{\rho_{2}} \alpha^{2} \lambda^{2} B-\frac{k \alpha}{\rho_{2}} \lambda A+\frac{k}{\rho_{2}} B-\frac{\delta \alpha}{\rho_{2}} \lambda C & =1 \\
i \lambda C+\frac{\alpha}{\rho_{3}} \lambda D+i \frac{\delta \alpha}{\rho_{3}} \lambda^{2} B & =0 \\
i \lambda D+\frac{\beta}{\tau} D-\frac{\alpha}{\tau} \lambda C & =0 .
\end{aligned}
$$

We have from (2.9)

$$
D=\frac{\alpha \lambda}{(i \tau \lambda+\beta)} C
$$

Combining (2.10) and (2.8) yields

$$
C=\frac{\lambda \delta \alpha(i \tau \lambda+\beta)}{i \alpha^{2} \lambda-\rho_{3}(i \tau \lambda+\beta)} B .
$$

On the other hand, by the definition of $\alpha$, we obtain from (2.6)

$$
B=-\frac{\rho_{1}}{k \alpha \lambda}
$$

Let $\Theta:=\frac{b \rho_{1}}{\rho_{2} k}-1$. Then, using (2.11) and (2.12) in (2.7) we have

$$
\Theta \lambda^{2} B+\frac{k}{\rho_{2}} B-\frac{k \alpha}{\rho_{2}} \lambda A-\frac{\lambda^{2} \delta^{2} \alpha^{2}(i \tau \lambda+\beta)}{\left[i \alpha^{2} \lambda-\rho_{3}(i \tau \lambda+\beta)\right] \rho_{2}} B=1 .
$$

Using (2.12) in (2.13) results in

$$
\frac{k \alpha \lambda}{\rho_{2}} A=-\Theta \lambda \frac{\rho_{1}}{k \alpha}-\frac{\rho_{1}}{\rho_{2} \alpha \lambda}-1+\frac{\lambda \delta^{2} \alpha(i \tau \lambda+\beta) \rho_{1}}{\left[i \alpha^{2} \lambda-\rho_{3}(i \tau \lambda+\beta)\right] \rho_{2} k}
$$

that is, using $\alpha=\sqrt{\frac{\rho_{1}}{k}}$,

$$
A=-\Theta \frac{\rho_{2}}{k}-\frac{1}{\lambda^{2}}-\frac{\rho_{2}}{\sqrt{\rho_{1} k} \lambda}+P(\lambda)
$$

where

$$
P(\lambda):=\frac{\delta^{2}(i \tau \lambda+\beta) \rho_{1}}{\left[i \alpha^{2} \lambda-\rho_{3}(i \tau \lambda+\beta)\right] k^{2}} \quad \text { with } \quad \lim _{\lambda \rightarrow \infty} \lambda|P(\lambda)|=\infty .
$$

Recalling that $v^{2}=i \lambda v^{1}=i \lambda A \cos (\alpha \lambda x)$ we get

$$
v^{2}(x)=\left(-\Theta \frac{i \lambda \rho_{2}}{k}-\frac{i}{\lambda}-\frac{i \rho_{2}}{\sqrt{\rho_{1} k}}+i \lambda P(\lambda)\right) \cos (\alpha \lambda x) .
$$


Note that

$$
\begin{aligned}
\left\|v^{2}\right\|_{L^{2}} & =\left(\int_{0}^{L}\left|v^{2}\right|^{2} d x\right)^{1 / 2} \\
& =\frac{\sqrt{L}}{2}\left|-\Theta \frac{\lambda \rho_{2}}{k}-\frac{1}{\lambda}-\frac{\rho_{2}}{\sqrt{\rho_{1} k}}+\lambda P(\lambda)\right| \\
& \geq-\underbrace{\frac{\sqrt{L}}{2}\left|\frac{1}{\lambda}+\frac{\rho_{2}}{\sqrt{\rho_{1} k}}\right|}_{\text {bounded as } \lambda \rightarrow \infty}+\frac{\sqrt{L}}{2}\left|P(\lambda)-\left(\frac{\rho_{2}}{b}-\frac{\rho_{1}}{k}\right) \frac{b}{k}\right| \lambda
\end{aligned}
$$

which implies (even) for $\frac{\rho_{1}}{k}=\frac{\rho_{2}}{b}$

$$
\lim _{\lambda \rightarrow \infty}\left\|U_{n}\right\|_{\mathcal{H}_{1}} \geq \lim _{\lambda \rightarrow \infty}\left\|v^{2}\right\|_{L^{2}}=\infty
$$

Thus we have proved

Theorem 2.2 The Timoshenko system (2.1)-(2.3) is not exponentially stable under Cattaneo's law, (even) under the assumption (2.4) - in contrast to the situation with the Fourier law $(\tau=0)$.

Remark. We mention that we could have a similar statement for the following set of boundary conditions replacing (2.3),

$$
\varphi_{x}(\cdot, 0)=\varphi_{x}(\cdot, L)=\psi(\cdot, 0)=\psi(\cdot, L)=q(\cdot, 0)=q(\cdot, L)=0 \quad \text { in }(0, \infty)
$$

cp. Sections 3 and 4, where we shall deal with this boundary condition to demonstrate that all arguments mutatis mutandis apply to both set of boundary conditions.

\section{Timoshenko with history — non-exponential stability for Cat- taneo's law}

Here we consider the Timoshenko system (1.13)-(1.16) with history and the Cattaneo law $(\tau>$ 0 ), and we prove that it is not exponentially stable even if we assume (1.12),

$$
\frac{\rho_{1}}{k}=\frac{\rho_{2}}{b} .
$$

First we again give a reformulation as first-order evolution system. The second-order differential equations are

$$
\begin{aligned}
\rho_{1} \varphi_{t t}-k\left(\varphi_{x}+\psi\right)_{x} & =0 \\
\rho_{2} \psi_{t t}-b \psi_{x x}+\int_{0}^{\infty} g(s) \psi_{x x}(x, t-s) d s+k\left(\varphi_{x}+\psi\right)+\delta \theta_{x} & =0 \\
\rho_{3} \theta_{t}+q_{x}+\delta \psi_{x t} & =0 \\
\tau q_{t}+\beta q+\theta_{x} & =0 .
\end{aligned}
$$

Let

$$
\eta(t, s, x):=\psi(t, x)-\psi(t-s, x), \quad t, s \geq 0
$$


then we have

$$
\begin{aligned}
\rho_{2} \psi_{t t}-\left(b-\int_{0}^{\infty} g(s) d s\right) \psi_{t t}-k\left(\varphi_{x}+\psi\right)_{x} & =0 \\
\rho_{3} \theta_{t}+q_{x}+\delta \psi_{x t} & =0 \\
\tau q_{t}+\beta q+\theta_{x} & =0 \\
\eta_{t}+\eta_{s}-\psi_{t} & =0 \\
\eta(\cdot, 0, \cdot) & =0
\end{aligned}
$$

where equation (3.8) is obtained differentiating (3.3). The initial conditions are given by

$$
\begin{aligned}
& \varphi(0, \cdot)=\varphi_{0}, \varphi_{t}(0, \cdot)=\varphi_{1}, \psi(0, \cdot)=\psi_{0}, \psi_{t}(0, \cdot)=\psi_{1}, \theta(0, \cdot)=\theta_{0}, \\
& q(0, \cdot)=q_{0}, \eta(0, s, \cdot)=\psi_{0}-\psi(-s, \cdot)=: \eta_{0}(s, \cdot) \quad \text { in }(0, L), s \geq 0,
\end{aligned}
$$

where the history is considered as an initial value. The boundary conditions are given by

$$
\varphi_{x}(\cdot, 0)=\varphi_{x}(\cdot, L)=\psi(\cdot, 0)=\psi(\cdot, L)=q(\cdot, 0)=q(\cdot, L)=0 \quad \text { in }(0, \infty) .
$$

Concerning the kernel $g$ we assume the following hypotheses $(t \geq 0)$,

$$
\begin{gathered}
g(t)>0, \quad \exists k_{0}, k_{1}, k_{2}>0:-k_{0} g(t) \leq g^{\prime}(t) \leq-k_{1} g(t), \quad\left|g^{\prime \prime}(t)\right| \leq k_{2} g(t) \\
\tilde{b}:=b-\int_{0}^{\infty} g(s) d s>0 .
\end{gathered}
$$

Remark. The associated energy term is given by

$$
E(t):=\frac{1}{2} \int_{0}^{L}\left[\rho_{1} \varphi_{t}^{2}+\rho_{2} \psi_{t}^{2}+\tilde{b} \psi_{x}^{2}+k\left|\varphi_{x}+\psi\right|^{2}+\rho_{3} \theta^{2}+\tau q^{2}+\int_{0}^{\infty} g(s)\left|\eta_{x}\right|^{2} d s\right] d x
$$

which is reflected in the norm of $\|U(t)\|_{\mathcal{H}}$ in the semigroup formulation now following. Let

$$
U:=\left(\varphi, \varphi_{t}, \psi, \psi_{t}, \theta, q, \eta\right)^{\prime}:=\left(u^{1}, u^{2}, u^{3}, u^{4}, u^{5}, u^{6}, u^{7}\right)^{\prime} .
$$

Then we formally have

$$
U_{t}=\mathcal{A}_{2} U, \quad U(0)=U_{0}
$$

where $U_{0}:=\left(\varphi_{0}, \varphi_{1}, \psi_{0}, \psi_{1}, \theta_{0}, q_{0}, \eta_{0}\right)^{\prime}$ and $\mathcal{A}_{2}$ is the formal differential operator

$$
\mathcal{A}_{2}:=\left(\begin{array}{ccccccc}
0 & I d & 0 & 0 & 0 & 0 & 0 \\
\frac{k}{\rho_{1}} \partial_{x}^{2} & 0 & \frac{k}{\rho_{1}} \partial_{x} & 0 & 0 & 0 & 0 \\
0 & 0 & 0 & I d & 0 & 0 & 0 \\
-\frac{k}{\rho_{2}} \partial_{x} & 0 & \frac{\tilde{b}}{\rho_{2}} \partial_{x}^{2}-\frac{k}{\rho_{2}} I d & 0 & -\frac{\delta}{\rho_{2}} \partial_{x} & 0 & \frac{1}{\rho_{2}} \int_{0}^{\infty} g(s) \partial_{x}^{2}(\cdot, s) d s \\
0 & 0 & 0 & -\frac{\delta}{\rho_{3}} \partial_{x} & 0 & -\frac{1}{\rho_{3}} \partial_{x} & 0 \\
0 & 0 & 0 & 0 & -\frac{1}{\tau} \partial_{x} & -\frac{\beta}{\tau} I d & 0 \\
0 & 0 & 0 & I d & 0 & 0 & -\partial_{s}
\end{array}\right) .
$$


Let

$$
\mathcal{H}_{2}=H_{*}^{1}(0, L) \times L_{*}^{2}(0, L) \times H_{0}^{1}(0, L) \times L^{2}(0, L) \times L_{*}^{2}(0, L) \times L^{2}(0, L) \times L_{g}^{2}\left(\mathbb{R}^{+}, H_{0}^{1}\right)
$$

where $L_{g}^{2}\left(\mathbb{R}^{+}, H_{0}^{1}\right)$ denotes the Hilbert space of $H_{0}^{1}$-valued functions on $\mathbb{R}^{+}$, endowed with the inner product

$$
\langle\varphi, \psi\rangle_{L_{g}^{2}\left(\mathbb{R}^{+}, H_{0}^{1}\right)}=\int_{0}^{L} \int_{0}^{\infty} g(s) \varphi_{x}(s, x) \psi_{x}(s, x) d s d x .
$$

Then $\mathcal{H}_{2}$, with norm

$$
\begin{aligned}
\|U\|_{\mathcal{H}_{2}}^{2}= & \rho_{1}\left\|u^{2}\right\|_{L^{2}}^{2}+\rho_{2}\left\|u^{4}\right\|_{L^{2}}^{2}+\tilde{b}\left\|u_{x}^{3}\right\|_{L^{2}}^{2}+k\left\|u_{x}^{1}+u^{3}\right\|_{L^{2}}^{2}+\rho_{3}\left\|u^{5}\right\|_{L^{2}}^{2} \\
& +\tau\left\|u^{6}\right\|_{L^{2}}^{2}+\left\|u^{7}\right\|_{L_{g}^{2}\left(\mathbb{R}^{+}, H_{0}^{1}\right)}^{2}
\end{aligned}
$$

is a Hilbert space. The domain of the operator $\mathcal{A}_{2}$ is now given by

$$
\begin{gathered}
D\left(\mathcal{A}_{2}\right):=\left\{U \in \mathcal{H}_{2} \mid u^{1} \in H^{2}(0, L), u_{x}^{1} \in H_{0}^{1}(0, L), u^{2} \in H_{*}^{1}(0, L), u^{4} \in H_{0}^{1}(0, L),\right. \\
u^{5} \in H_{*}^{1}(0, L), u^{6} \in H_{0}^{1}(0, L), \tilde{b} u^{3}+\int_{0}^{\infty} g(s) u^{7}(s, \cdot) d s \in H^{2}(0, L) \cap H_{0}^{1}(0, L), \\
\left.u_{s}^{7} \in L_{g}^{2}\left(\mathbb{R}^{+}, H_{0}^{1}\right), u^{7}(0, x)=0(x \in(0, L))\right\} .
\end{gathered}
$$

We shall prove

Lemma 3.1 The operator $\mathcal{A}_{2}$ is the infinitesimal generator of a $C_{0}$-semigroup of contractions.

Proof. First we note that $\mathcal{A}_{2}$ is dissipative, because for any $U \in D\left(\mathcal{A}_{2}\right)$ we have

$$
\begin{aligned}
\operatorname{Re}\left\langle\mathcal{A}_{2} U, U\right\rangle_{\mathcal{H}_{2}} & =\frac{1}{2} \int_{0}^{L} \int_{0}^{\infty} g^{\prime}(s)\left|u_{x}^{7}\right|^{2} d s d x-\beta \int_{0}^{L}\left|u^{6}\right|^{2} d x \\
& \leq-\frac{k_{1}}{2} \int_{0}^{L} \int_{0}^{L} g(s)\left|u_{x}^{7}\right|^{2} d s d x-\beta \int_{0}^{L}\left|u^{6}\right|^{2} d x \leq 0 .
\end{aligned}
$$

Now we show that $0 \in \varrho\left(\mathcal{A}_{2}\right)$. For any $F=\left(f^{1}, f^{2}, f^{3}, f^{4}, f^{5}, f^{6}, f^{7}\right)^{\prime} \in \mathcal{H}_{2}$, consider the following equation,

$$
\mathcal{A}_{2} U=F
$$

that is,

$$
\begin{aligned}
u^{2} & =f^{1} \\
k\left(u_{x}^{1}+u^{3}\right)_{x} & =\rho_{1} f^{2} \\
u^{4} & =f^{3} \\
\tilde{b} u_{x x}^{3}+\int_{0}^{\infty} g(s) u_{x x}^{7}(\cdot, s) d s-k\left(u_{x}^{1}+u^{3}\right)-\delta u_{x}^{5} & =\rho_{2} f^{4} \\
-u_{x}^{6}-\delta u_{x}^{4} & =\rho_{3} f^{5} \\
-\beta u^{6}-u_{x}^{5} & =\tau f^{6} \\
-u_{s}^{7}+u^{4} & =f^{7} .
\end{aligned}
$$


From (3.17) and (3.19) we can get a unique $u^{2} \in H_{*}^{1}(0, L)$ and $u^{4} \in H_{0}^{1}(0, L)$, respectively. Then, from (3.21) we have

$$
u^{6}=-\delta u^{4}-\rho_{3} \int_{0}^{x} f^{5}(y) d y
$$

where $u^{6}(0)=u^{6}(L)=0$, that is we get a unique $u^{6} \in H_{0}^{1}(0, L)$. Also, from (3.23), we can determine

$$
u^{7}=s u^{4}-\int_{0}^{s} f^{7}(\xi) d \xi
$$

It is clear that $u^{7}(0, \cdot)=0$ and $u_{s}^{7} \in L_{g}^{2}\left(\mathbb{R}^{+}, H_{0}^{1}\right)$. To prove that $u^{7} \in L_{g}^{2}\left(\mathbb{R}^{+}, H_{0}^{1}\right)$, let $T, \epsilon>0$ be arbitrary. Using (3.12) we have

$$
\begin{aligned}
\int_{\epsilon}^{T} \mid g(s)\|\| u_{x}^{7} \|_{L^{2}}^{2} d s & \leq-\frac{1}{k_{1}} \int_{\epsilon}^{T} g^{\prime}(s)\left\|u_{x}^{7}\right\|_{L^{2}}^{2} d s \\
& \leq-\frac{1}{k_{1}} g(T)\left\|u_{x}^{7}(T)\right\|_{L^{2}}^{2}+\frac{1}{k_{1}} g(\epsilon)\left\|u_{x}^{7}(\epsilon)\right\|_{L^{2}}^{2}+\frac{2}{k_{1}} \int_{\epsilon}^{T} g(s)\left\langle u_{x}^{7}(s), u_{x s}^{7}(s)\right\rangle_{L^{2}} d s \\
& \leq \frac{1}{k_{1}} g(\epsilon)\left\|u_{x}^{7}(\epsilon)\right\|_{L^{2}}^{2}+\frac{1}{2} \int_{\epsilon}^{T} g(s)\left\|u_{x}^{7}\right\|_{L^{2}}^{2} d s+\frac{2}{k_{1}^{2}} \int_{\epsilon}^{T} g(s)\left\|u_{x s}^{7}\right\|_{L^{2}}^{2} d s
\end{aligned}
$$

that is

$$
\int_{\epsilon}^{T}|g(s)|\left\|u_{x}^{7}\right\|_{L^{2}}^{2} d s \leq \frac{2}{k_{1}} g(\epsilon)\left\|u_{x}^{7}(\epsilon)\right\|_{L^{2}}^{2}+\frac{4}{k_{1}^{2}} \int_{\epsilon}^{T} g(s)\left\|u_{x s}^{7}\right\|_{L^{2}}^{2} d s .
$$

Since using the hypotheses on $g$ and the properties of $u^{7}$, we have

$$
\frac{1}{k_{1}} g(\epsilon)\left\|u_{x}^{7}(\epsilon)\right\|_{L^{2}}^{2} \longrightarrow 0 \quad \text { as } \quad \epsilon \rightarrow 0
$$

we obtain from (3.24), letting $T \rightarrow \infty$ and $\epsilon \rightarrow 0$,

$$
\left\|u^{7}\right\|_{L_{g}^{2}}^{2} \leq \frac{4}{k_{1}^{2}} \int_{0}^{\infty} g(s)\left\|u_{x s}^{7}\right\|_{L^{2}}^{2} d s<\infty .
$$

Therefore $u^{7} \in L_{g}^{2}\left(\mathbb{R}^{+} ; H_{0}^{1}\right)$. On the other hand, from (3.22) we have that $u^{5}$ is uniquely given by

$$
u^{5}=-\int_{0}^{x}\left(\beta u^{6}(y)+\tau f^{6}(y)\right) d y+\frac{1}{L} \int_{0}^{L} \int_{0}^{x}\left(\beta u^{6}(y)+\tau f^{6}(y)\right) d y d x
$$

that is, $u^{5} \in H_{*}^{1}(0, L)$. Also, from (3.18) we have that

$$
u_{x}^{1}+u^{3}=\frac{\rho_{1}}{k} \int_{0}^{x} f^{2}(y) d y \in H_{0}^{1}(0, L)
$$


then from $(3.20)$

$$
\tilde{b} u_{x x}^{3}+\int_{0}^{\infty} g(s) u_{x x}^{7}(\cdot, s) d s=G
$$

where $G:=k\left(u_{x}^{1}+u^{3}\right)+\delta u_{x}^{5}+\rho_{2} f^{4} \in L^{2}(0, L)$. By standard elliptic theory we obtain a unique

$$
\tilde{b} u^{3}+\int_{0}^{\infty} g(s) u^{7}(\cdot, s) d s \in H^{2}(0, L) \cap H_{0}^{1}(0, L)
$$

satisfying (3.26). Since $u^{7} \in L_{g}^{2}\left(\mathbb{R}^{+} ; H_{0}^{1}\right)$, we conclude from the last equation that $u^{3} \in H_{0}^{1}(0, L)$. Again from (3.25) we can get a unique $u^{1} \in H^{2}(0, L) \cap H_{*}^{1}(0, L)$ such that $u_{x}^{1} \in H_{0}^{1}(0, L)$. Thus the unique solvability of (3.16) with $U=\left(u^{1}, u^{2}, u^{3}, u^{4}, u^{5}, u^{6}, u^{7}\right)^{\prime} \in D\left(\mathcal{A}_{2}\right)$ is proved. Moreover, it is now obvious that there is a positive constant $K$, being independent of $U$, such that

$$
\|U\|_{\mathcal{H}_{2}} \leq K\|F\|_{\mathcal{H}_{2}} .
$$

This implies that $0 \in \varrho\left(\mathcal{A}_{2}\right)$. Since $\mathcal{A}_{2}$ is dissipative, it follows that $\mathcal{A}_{2}$ is the infinitesimal generator of a contraction semigroup in $\mathcal{H}_{2}$.

$$
\text { Q.E.D. }
$$

Finally we show that the original second-order system (3.2) and the evolution equation (3.15), using the transformations (3.3), (3.14), are fully equivalent. In fact, it is clear by construction that the solution of the system (3.2), with the notation (3.14), satisfies (3.15). On other hand, let $U=\left(u^{1}, u^{2}, u^{3}, u^{4}, u^{5}, u^{6}, u^{7}\right)^{\prime}$ be the solution to (3.15). Then we conclude

$$
\begin{aligned}
u_{t}^{1} & =u^{2} \\
u_{t}^{3} & =u^{4} \\
\rho_{1} u_{t t}^{1}-k\left(u_{x}^{1}+u^{3}\right)_{x} & =0 \\
\rho_{2} u_{t t}^{3}-\tilde{b} u_{x x}^{3}-\int_{0}^{\infty} g(s) u_{x x}^{7}(x, s) d s+k\left(u_{x}^{1}+u^{3}\right)+\delta u_{x}^{5} & =0 \\
\rho_{3} u_{t}^{5}+u_{x}^{6}+\delta u_{x t}^{3} & =0 \\
\tau u_{t}^{6}+\beta u^{6}+u_{x}^{5} & =0 \\
u_{t}^{7}+u_{s}^{7}-u_{t}^{3} & =0 .
\end{aligned}
$$

Therefore, $\left(u^{1}, u^{3}, u^{5}, u^{6}, u^{7}\right)^{\prime}$ is a solution of the system (3.4)-(3.8). Then, by uniqueness of solutions,

$$
\left(u^{1}, u^{3}, u^{5}, u^{6}, u^{7}\right)=(\varphi, \psi, \theta, q, \eta) \in H_{*}^{1}(0, L) \times H_{0}^{1}(0, L) \times L_{*}^{2}(0, L) \times L^{2}(0, L) \times L_{g}^{2}\left(\mathbb{R}^{+}, H_{0}^{1}\right)
$$

with condition $\eta(\cdot, 0, \cdot)=0$, and, using $(3.27),(3.28)$, we have that the solution $U$ of the evolution problem (3.15) is also a solution of (3.4)-(3.9). Then the evolution equation (3.15) is fully equivalent to the system (3.4)-(3.9). That $\eta$ satisfies the equality (3.3) easily follows observing that the characteristic lines of equation (3.29) are given by $\Gamma(s)=\left(\Gamma^{1}(s), \Gamma^{2}(s)\right)=(s, s+c)$, where $c$ is a constant. Therefore, using (3.28), we have

$$
\eta_{s}(\Gamma(s), \cdot)=\psi_{t}\left(\Gamma^{2}(s), \cdot\right) .
$$


By integration over $[0, s]$ and using $\eta(\cdot, 0, \cdot)=0$ we have $\eta(s, s+c, \cdot)=\psi(s+c, \cdot)-\psi(c, \cdot)$. Then, putting $c=t-s$ our conclusion on the equivalence of (3.2) and (3.15) follows.

Now we are going to prove that the system is not exponentially stable, where we shall need the following Lemma from [3], cp. [4].

Lemma 3.2 Let us suppose that $g$ satisfies the conditions (3.12) and let us assume that

$$
\lim _{s \rightarrow 0} \sqrt{s} g(s)=0 .
$$

Then there exists $C>0$ such that

$$
\left|\lambda \int_{0}^{\infty} g(s) e^{-i \lambda s} d s\right| \leq C
$$

uniformly in $\lambda \in \mathbb{R}$.

As in Section 2, using Lemma 2.1, to show the non-exponential stability it is sufficient to find sequences $\left(\lambda_{n}\right)_{n} \subset \mathbb{R}$ with $\lim _{n \rightarrow \infty}\left|\lambda_{n}\right|=\infty$, and $\left(U_{n}\right)_{n} \subset D\left(\mathcal{A}_{2}\right),\left(F_{n}\right)_{n} \subset \mathcal{H}_{2}$, such that $\left(i \lambda_{n} I d-\mathcal{A}_{2}\right) U_{n}=F_{n}$ is bounded and

$$
\lim _{n \rightarrow \infty}\left\|U_{n}\right\|_{\mathcal{H}_{2}}=\infty
$$

As $F_{n} \equiv F$ we choose $F:=(0, \cos (\alpha \lambda x), 0, \sin (\alpha \lambda x), 0,0,0)^{\prime}$, where

$$
\lambda \equiv \lambda_{n}:=\frac{n \pi}{\alpha L} \quad(n \in \mathbb{N}), \quad \alpha:=\sqrt{\frac{\rho_{1}}{k}} .
$$

The solution $U \equiv\left(v^{1}, v^{2}, v^{3}, v^{4}, v^{5}, v^{6}, v^{7}\right)^{\prime}$ to $\left(i \lambda I d-\mathcal{A}_{2}\right) U=F$, should satisfy

$$
\begin{aligned}
i \lambda v^{1}-v^{2} & =0 \\
i \lambda v^{3}-v^{4} & =0 \\
-\lambda^{2} v^{1}-\frac{k}{\rho_{1}} v_{x x}^{1}-\frac{k}{\rho_{1}} v_{x}^{3} & =f^{2} \\
-\lambda^{2} v^{3}-\frac{b}{\rho_{2}} v_{x x}^{3}+\frac{b_{0}}{\rho_{2}} v_{x x}^{3}-\frac{1}{\rho_{2}} \int_{0}^{\infty} g(s) v_{x x}^{7}(x, s) d s+\frac{k}{\rho_{2}} v_{x}^{1}+\frac{k}{\rho_{2}} v^{3}+\frac{\delta}{\rho_{2}} v_{x}^{5} & =f^{4} \\
i \lambda v^{5}+\frac{1}{\rho_{3}} v_{x}^{6}+i \lambda \frac{\delta}{\rho_{3}} v_{x}^{3} & =0 \\
i \lambda v^{6}+\frac{\beta}{\tau} v^{6}+\frac{1}{\tau} v_{x}^{5} & =0 \\
i \lambda v^{7}+v_{s}^{7}-i \lambda v^{3} & =0 .
\end{aligned}
$$

where $b_{0}:=\int_{0}^{\infty} g(s) d s$. This can be solved by

$$
\begin{gathered}
v^{1}(x)=A \cos (\alpha \lambda x), \quad v^{3}(x)=B \sin (\alpha \lambda x), \\
v^{5}(x)=C \cos (\alpha \lambda x), \quad v^{6}(x)=D \sin (\alpha \lambda x), \quad v^{7}(x, s)=\varphi(s) \sin (\alpha \lambda x)
\end{gathered}
$$


where $A, B, C, D, \varphi(s)$ depend on $\lambda$ and will be determined explicitly in the sequel. Note that this choose is just compatible with the boundary conditions. System (3.30) is equivalent to

$$
\begin{gathered}
-\lambda^{2} A+\frac{k}{\rho_{1}} \alpha^{2} \lambda^{2} A-\frac{k}{\rho_{1}} \alpha \lambda B=1 \\
-\lambda^{2} B+\frac{b}{\rho_{2}} \alpha^{2} \lambda^{2} B-\frac{b_{0}}{\rho_{2}} \alpha^{2} \lambda^{2} B+\frac{\alpha^{2} \lambda^{2}}{\rho_{2}} \int_{0}^{\infty} g(s) \varphi(s) d s-\frac{k}{\rho_{2}} \alpha \lambda A+\frac{k}{\rho_{2}} B-\frac{\delta \alpha}{\rho_{2}} \lambda C=1 \\
i \lambda C+\frac{\alpha}{\rho_{3}} \lambda D+i \frac{\delta \alpha}{\rho_{3}} \lambda^{2} B=0 \\
i \lambda D+\frac{\beta}{\tau} D-\frac{\alpha}{\tau} \lambda C=0 \\
i \lambda \varphi(s)+\varphi^{\prime}(s)-i \lambda B=0 .
\end{gathered}
$$

From (3.34) we have

$$
D=\frac{\alpha \lambda}{(i \tau \lambda+\beta)} C
$$

Combining (3.36) and (3.33) we get

$$
C=\frac{\lambda \delta \alpha(i \tau \lambda+\beta)}{i \alpha^{2} \lambda-\rho_{3}(i \tau \lambda+\beta)} B .
$$

On the other hand, by the definition of $\alpha=\sqrt{\frac{\rho_{1}}{k}}$, we obtain from (3.31)

$$
B=-\frac{\rho_{1}}{k \alpha \lambda} \text {. }
$$

Since $\eta(\cdot, 0, \cdot)=0$ we have that $\varphi(0)=0$, then solving (3.35) we get

$$
\varphi(s)=B-B e^{-i \lambda s} .
$$

From (3.39) we get

$$
\int_{0}^{\infty} g(s) \varphi(s) d s=\int_{0}^{\infty} g(s)\left[B-B e^{-i \lambda s}\right] d s=B b_{0}-B \int_{0}^{\infty} g(s) e^{-i \lambda s} d s .
$$

Let $\Theta:=\frac{b \rho_{1}}{\rho_{2} k}-1$. Then, using (3.37),(3.38) and (3.40) in (3.32) we obtain

$$
\frac{k \alpha \lambda}{\rho_{2}} A=-\Theta \lambda \alpha+\frac{\alpha^{3} \lambda}{\rho_{2}}\left(\int_{0}^{\infty} g(s) e^{-i \lambda s} d s\right)-\frac{k \alpha}{\rho_{2} \lambda}-1+\frac{\lambda \delta^{2} \alpha^{3}(i \tau \lambda+\beta)}{\left[i \alpha^{2} \lambda-\rho_{3}(i \tau \lambda+\beta)\right] \rho_{2}}
$$

that is, using $\alpha=\sqrt{\frac{\rho_{1}}{k}}$,

$$
A=-\Theta \frac{\rho_{2}}{k}+\frac{\alpha^{2}}{k}\left(\int_{0}^{\infty} g(s) e^{-i \lambda s} d s\right)-\frac{\rho_{2}}{k \alpha \lambda}-\frac{1}{\lambda^{2}}+P(\lambda)
$$

where

$$
P(\lambda):=\frac{\delta^{2}(i \tau \lambda+\beta) \rho_{1}}{\left[i \alpha^{2} \lambda-\rho_{3}(i \tau \lambda+\beta)\right] k^{2}} \quad \text { with } \quad \lim _{\lambda \rightarrow \infty} \lambda|P(\lambda)|=\infty
$$


Remark. We observe that to conclude (3.41) it is essential that the coupling parameter $\delta$ is different from zero.

Recalling that $v^{2}=i \lambda v^{1}=i \lambda A \cos (\delta \lambda x)$ we get

$$
v^{2}(x)=\left(-i \lambda \frac{\rho_{2}}{k} \Theta+\frac{i \rho_{1}}{k^{2}} \lambda \int_{0}^{\infty} g(s) e^{-i \lambda s} d s-\frac{i \rho_{2}}{k \alpha}-\frac{i}{\lambda}+i \lambda P(\lambda)\right) \cos (\delta \lambda x) .
$$

Note that

$$
\begin{aligned}
\left\|v^{2}\right\|_{L^{2}} & =\left(\int_{0}^{L}\left|v^{2}\right|^{2} d x\right)^{1 / 2} \\
& =\frac{\sqrt{L}}{2}\left|-\lambda \frac{\rho_{2}}{k} \Theta+\frac{\rho_{1}}{k^{2}} \lambda \int_{0}^{\infty} g(s) e^{-i \lambda s} d s-\frac{\rho_{2}}{k \alpha}-\frac{1}{\lambda}+\lambda P(\lambda)\right| \\
& \geq \underbrace{-\frac{\sqrt{L}}{2}\left|-\frac{1}{\lambda}-\frac{\rho_{2}}{\sqrt{\rho_{1} k}}+\frac{\rho_{1}}{k^{2}} \lambda \int_{0}^{\infty} g(s) e^{-i \lambda s} d s\right|}_{\text {bounded as } \lambda \rightarrow \infty}+\frac{\sqrt{L}}{2}\left|P(\lambda)-\left(\frac{\rho_{2}}{b}-\frac{\rho_{1}}{k}\right) \frac{b}{k}\right| \lambda
\end{aligned}
$$

and using Lemma 3.2, we get

$$
\lim _{\lambda \rightarrow \infty}\left\|U_{n}\right\|_{\mathcal{H}_{2}}^{2} \geq \lim _{\lambda \rightarrow \infty}\left\|v^{2}\right\|_{L^{2}}^{2}=\infty
$$

which completes our conclusion summarized in

Theorem 3.3 The Timoshenko system with history (3.2),(3.10)-(3.11) is not exponentially stable under Cattaneo's law, (even) under the assumption (3.1).

This result is in contrast to the exponential stability under the Fourier law, assuming (3.1), which we shall prove in the last section. The more it is interesting to notice that it also contrasts the known (see [3], cp. [1] for finite history) exponential stability for the case that there is no heat conduction. This means that the Fourier model of heat conduction preserves the exponential stability of the model, while the - still assumed to have a dissipative effect - Cattaneo model destabilizes in the sense that it is no longer exponentially stable.

Technically in the proof, this effect can be seen in (3.41), see the remark following there.

\section{Timoshenko with history — exponential stability for Fourier's law}

Here we consider the Timoshenko system (1.13)-(1.16) with history and the Fourier law $(\tau=0)$, and we prove that it is exponentially stable if and only if (1.12) holds. For $\tau=0$ we can elimate $q$ easily and obtain the following differential equation for $\theta$,

$$
\rho_{3} \theta_{t}-\tilde{\beta} \theta_{x x}+\delta \psi_{x t}=0
$$


where $\tilde{\beta}:=\beta^{-1}>0$. Then, introducing $\eta$ as in the previous section in (3.3), we have the differential equations

$$
\begin{aligned}
\rho_{1} \varphi_{t t}-k\left(\varphi_{x}+\psi\right)_{x} & =0 \\
\rho_{2} \psi_{t t}-\left(b-\int_{0}^{\infty} g(s) d s\right) \psi_{x x}-\int_{0}^{\infty} g(s) \eta_{x x}(x, s) d s+k\left(\varphi_{x}+\psi\right)+\delta \theta_{x} & =0 \\
\rho_{3} \theta_{t}-\tilde{\beta} \theta_{x x}+\delta \psi_{x t} & =0 \\
\eta_{t}+\eta_{s}-\psi_{t} & =0
\end{aligned}
$$

with inital conditions

$$
\begin{gathered}
\varphi(0, \cdot)=\varphi_{0}, \varphi_{t}(0, \cdot)=\varphi_{1}, \psi(0, \cdot)=\psi_{0}, \psi_{t}(0, \cdot)=\psi_{1}, \theta(0, \cdot)=\theta_{0}, \\
q(0, \cdot)=q_{0}, \eta(0, s, \cdot)=\psi_{0}-\psi(-s, \cdot)=: \eta_{0}(s, \cdot) \quad \text { in }(0, L) s \geq 0
\end{gathered}
$$

and boundary conditions

$$
\varphi_{x}(\cdot, 0)=\varphi_{x}(\cdot, L)=\psi(\cdot, 0)=\psi(\cdot, L)=\theta_{x}(\cdot, 0)=\theta_{x}(\cdot, L)=0 \quad \text { in }(0, \infty) .
$$

Again transforming to a first-order system we obtain for

$$
\begin{gathered}
U:=\left(\varphi, \varphi_{t}, \psi, \psi_{t}, \theta, \eta\right)^{\prime} \equiv\left(u^{1}, u^{2}, u^{3}, u^{4}, u^{5}, u^{6}\right)^{\prime} \\
U_{t}=\mathcal{A}_{3} U, \quad U(0)=U_{0}
\end{gathered}
$$

where $\mathcal{A}_{3}$ is formally given by

$$
\mathcal{A}_{3}:=\left(\begin{array}{cccccc}
0 & I d & 0 & 0 & 0 & 0 \\
\frac{k}{\rho_{1}} \partial_{x}^{2} & 0 & \frac{k}{\rho_{1}} \partial_{x} & 0 & 0 & 0 \\
0 & 0 & 0 & I d & 0 & 0 \\
-\frac{k}{\rho_{2}} \partial_{x} & 0 & \frac{\tilde{b}}{\rho_{2}} \partial_{x}^{2}-\frac{k}{\rho_{2}} I d & 0 & -\frac{\delta}{\rho_{2}} \partial_{x} & \frac{1}{\rho_{2}} \int_{0}^{\infty} g(s) \partial_{x}^{2}(s, \cdot) d s \\
0 & 0 & 0 & -\frac{\delta}{\rho_{3}} \partial_{x} & \frac{\tilde{\beta}}{\rho_{3}} \partial_{x}^{2} & 0 \\
0 & 0 & 0 & I d & 0 & -\partial_{s}
\end{array}\right) .
$$

Let us denote by

$$
\mathcal{H}_{3}:=H_{*}^{1}(0, L) \times L_{*}^{2}(0, L) \times H_{0}^{1}(0, L) \times L^{2}(0, L) \times L_{*}^{2}(0, L) \times L_{g}^{2}\left(\mathbb{R}^{+}, H_{0}^{1}\right) .
$$

It is easy to see that $\mathcal{H}_{3}$ together with the norm

$$
\|U\|_{\mathcal{H}_{3}}^{2}=\rho_{1}\left\|u^{2}\right\|_{L^{2}}^{2}+\rho_{2}\left\|u^{4}\right\|_{L^{2}}^{2}+\tilde{b}\left\|u_{x}^{3}\right\|_{L^{2}}^{2}+k\left\|u_{x}^{1}+u^{3}\right\|_{L^{2}}^{2}+\rho_{3}\left\|u^{5}\right\|_{L^{2}}^{2}+\left\|u^{6}\right\|_{L_{g}^{2}\left(\mathbb{R}^{+}, H_{0}^{1}\right)}^{2}
$$

is a Hilbert space. The domain of the operator $\mathcal{A}_{3}$ is defined by

$$
\begin{gathered}
D\left(\mathcal{A}_{3}\right)=\left\{U \in \mathcal{H}_{3} \mid u^{1} \in H^{2}(0, L), u_{x}^{1} \in H_{0}^{1}(0, L), u^{2} \in H_{*}^{1}(0, L), u^{4} \in H_{0}^{1}(0, L),\right. \\
u_{x}^{5} \in H_{0}^{1}(0, L), \tilde{b} u^{3}+\int_{0}^{\infty} g(s) u^{6}(x, s) d s \in H^{2}(0, L) \cap H_{0}^{1}(0, L), \\
\left.u_{s}^{6} \in L_{g}^{2}\left(\mathbb{R}^{+}, H_{0}^{1}\right), u^{6}(0, x)=0, \quad(x \in(0, L))\right\} .
\end{gathered}
$$

As in Section 3 we can prove that $\mathcal{A}_{3}$, being dissipative with $0 \in \varrho\left(\mathcal{A}_{3}\right)$, generates a contraction semigroup. 


\subsection{Exponential stability for $\frac{\rho_{1}}{k}=\frac{\rho_{2}}{b}$}

In this subsection we will show that the system is exponentially stable infinity provided the condition

$$
\frac{\rho_{1}}{k}=\frac{\rho_{2}}{b}
$$

holds. Once more we use Lemma 2.1, and we have to check if the following two conditions hold,

$$
i \mathbb{R} \subset \varrho\left(\mathcal{A}_{3}\right)
$$

and

$$
\exists C>0 \forall \lambda \in \mathbb{R}: \quad\left\|\left(i \lambda I d-\mathcal{A}_{3}\right)^{-1}\right\|_{\mathcal{H}_{3}} \leq C .
$$

First we will show (4.2) using contradiction arguments. In fact, suppose that (4.2) is not true. Then (cp. [9, p.25]) there exists $\omega \in \mathbb{R}$, a sequence $\left(\beta_{n}\right)_{n} \subset \mathbb{R}$ with $\beta_{n} \rightarrow \omega,|\beta|<|\omega|$ and a sequence of functions

$$
U_{n}=\left(u_{n}^{1}, u_{n}^{2}, u_{n}^{3}, u_{n}^{4}, u_{n}^{5}, u_{n}^{6}\right)^{\prime} \in D\left(\mathcal{A}_{3}\right) \quad \text { with } \quad\left\|U_{n}\right\|_{\mathcal{H}_{3}}=1
$$

such that, as $n \rightarrow \infty$,

$$
i \beta_{n} U_{n}-\mathcal{A}_{3} U_{n} \longrightarrow 0 \quad \text { in } \quad \mathcal{H}_{3}
$$

that is,

$$
\begin{array}{ccc}
i \beta_{n} u_{n}^{1}-u_{n}^{2} \longrightarrow 0 & \text { in } H_{*}^{1}(0, L) & \\
i \beta_{n} \rho_{1} u_{n}^{2}-k\left(u_{n, x}^{1}+u_{n}^{3}\right)_{x} & \longrightarrow 0 & \text { in } L_{*}^{2}(0, L) \\
i \beta_{n} u_{n}^{3}-u_{n}^{4} \longrightarrow 0 & \text { in } H_{0}^{1}(0, L) & \\
i \beta_{n} \rho_{2} u_{n}^{4}-\tilde{b} u_{n, x x}^{3}-\int_{0}^{\infty} g(s) u_{n, x x}^{6}(\cdot, s) d s+k\left(u_{n, x}^{1}+u_{n}^{3}\right)+\delta u_{n, x}^{5} \longrightarrow 0 & \text { in } L^{2}(0, L) \\
i \beta_{n} \rho_{3} u_{n}^{5}-\tilde{\beta} u_{n, x x}^{5}+\delta u_{n, x}^{4} \longrightarrow 0 & \text { in } \quad L_{*}^{2}(0, L) \\
i \beta_{n} u_{n}^{6}+u_{n, s}^{6}-u_{n}^{4} \longrightarrow 0 & \text { in } \quad L_{g}^{2}\left(\mathbb{R}^{+}, H_{0}^{1}\right) .
\end{array}
$$

Taking the inner product of (4.5) with $U_{n}$ in $\mathcal{H}_{3}$ and then taking its real part yields

$$
-\operatorname{Re}\left\langle\mathcal{A}_{3} U_{n}, U_{n}\right\rangle_{\mathcal{H}_{3}}=-\frac{1}{2} \int_{0}^{L} \int_{0}^{\infty} g^{\prime}(s)\left|u_{n, x}^{6}\right|^{2} d s d x+\tilde{\beta} \int_{0}^{L}\left|u_{n, x}^{5}\right|^{2} d x \longrightarrow 0
$$

Using the hypotheses on $g$ we have that

$$
\begin{aligned}
& u_{n}^{6} \quad \longrightarrow \quad 0 \quad \text { in } \quad L_{g}^{2}\left(\mathbb{R}^{+}, H_{0}^{1}\right), \\
& u_{n}^{5} \quad \longrightarrow \quad 0 \quad \text { in } \quad H_{*}^{1}(0 ; L) \hookrightarrow L^{2}(0, L) .
\end{aligned}
$$

Then, using (4.4), we have that

$$
\rho_{1}|| u_{n}^{2}\left\|_{L^{2}}^{2}+\rho_{2}\right\| u_{n}^{4}\left\|_{L^{2}}^{2}+\tilde{\beta}\right\| u_{n, x}^{3}\left\|_{L^{2}}^{2}+k\right\| u_{n, x}^{1}+u_{n}^{3} \|_{L^{2}}^{2} \longrightarrow 1 .
$$


On the other hand, taking the inner product of (4.6) with $\rho_{1} u_{n}^{2}$ in $L_{*}^{2}(0, L)$ and (4.7) with $\rho_{1} u_{n}^{1}$ in $L_{*}^{2}(0, L)$, repectively, yields

$$
i \rho_{1} \beta_{n}\left(u_{n}^{1}, u_{n}^{2}\right)_{L^{2}}-\rho_{1}\left\|u_{n}^{2}\right\|_{L^{2}}^{2} \longrightarrow 0
$$

and

$$
i \rho_{1} \beta_{n}\left(u_{n}^{2}, u_{n}^{1}\right)_{L^{2}}-k\left(u_{n, x}^{1}+u_{n}^{3}, u_{n, x}^{1}\right)_{L^{2}} \longrightarrow 0 .
$$

Adding and taking the real part we get

$$
k R e\left(u_{n, x}^{1}+u_{n}^{3}, u_{n, x}^{1}\right)_{L^{2}}-\rho_{1}\left\|u_{n}^{2}\right\|_{L^{2}}^{2} \longrightarrow 0 .
$$

Analogously, taking the inner product of (4.8) with $\rho_{2} u_{n}^{4}$ in $L^{2}(0, L)$ and (4.9) with $u_{n}^{3}$ in $L^{2}(0, L)$, repectively, yields

$$
i \rho_{2} \beta_{n}\left(u_{n}^{3}, u_{n}^{4}\right)_{L^{2}}-\rho_{2}\left\|u_{n}^{4}\right\|_{L^{2}}^{2} \longrightarrow 0
$$

and

$$
\begin{aligned}
i \rho_{2} \beta_{n}\left(u_{n}^{4}, u_{n}^{3}\right)_{L^{2}} & +\tilde{b}\left\|u_{n, x}^{3}\right\|_{L^{2}}^{2}+\int_{0}^{\infty} g(s)\left(u_{n, x}^{6}, u_{n, x}^{3}\right)_{L^{2}} d s \\
& +k\left(u_{n, x}^{1}+u^{3}, u_{n}^{3}\right)_{L^{2}}+\delta\left(u_{n, x}^{5}, u_{n}^{3}\right)_{L^{2}} \longrightarrow 0 .
\end{aligned}
$$

Note that from (4.12), (4.13) we have

$$
\int_{0}^{\infty} g(s)\left(u_{n, x}^{6}, u_{n, x}^{3}\right)_{L^{2}} d s+\delta\left(u_{n, x}^{5}, u_{n}^{3}\right)_{L^{2}} \longrightarrow 0,
$$

this used in (4.17) results in

$$
i \rho_{2} \beta_{n}\left(u_{n}^{4}, u_{n}^{3}\right)_{L^{2}}+\tilde{b}\left\|u_{n, x}^{3}\right\|_{L^{2}}^{2}+k\left(u_{n, x}^{1}+u_{n}^{3}, u_{n}^{3}\right)_{L^{2}} \longrightarrow 0 .
$$

Adding (4.16) and (4.18) and taking real part, we get

$$
-\rho_{2}\left\|u_{n}^{4}\right\|_{L^{2}}^{2}+\tilde{b}|| u_{n, x}^{3} \|_{L^{2}}^{2}+k R e\left(u_{n, x}^{1}+u_{n}^{3}, u_{n}^{3}\right)_{L^{2}} \longrightarrow 0
$$

and adding (4.15) with (4.19) we have

$$
\tilde{b}\left\|u_{n, x}^{3}\right\|_{L^{2}}^{2}+k\left\|u_{n, x}^{1}+u_{n}^{3}\right\|_{L^{2}}^{2}-\rho_{1}\left\|u_{n}^{2}\right\|_{L^{2}}^{2}-\rho_{2}\left\|u_{n}^{4}\right\|_{L^{2}}^{2} \longrightarrow 0 .
$$

Consequently, from (4.14) and (4.20) we deduce that

$$
\begin{aligned}
\tilde{b}\left\|u_{n, x}^{3}\right\|_{L^{2}}^{2}+k\left\|u_{n, x}^{1}+u_{n}^{3}\right\|_{L^{2}}^{2} & \longrightarrow \frac{1}{2} \\
\rho_{1}\left\|u_{n}^{2}\right\|_{L^{2}}^{2}+\rho_{2}\left\|u_{n}^{4}\right\|_{L^{2}}^{2} & \longrightarrow \frac{1}{2} .
\end{aligned}
$$


Also, it is clear that $s \mapsto \frac{1}{\beta_{n}^{2}} u_{n}^{4} \in L_{g}^{2}\left(\mathbb{R}^{+} ; H_{0}^{1}\right)$. Then multiplying (4.11) with $\frac{1}{\beta_{n}^{2}} u_{n}^{4}$ in $L_{g}^{2}\left(\mathbb{R}^{+} ; H_{0}^{1}\right)$ gives

$$
i\left(u_{n}^{6}, \frac{u_{n}^{4}}{\beta_{n}}\right)_{L_{g}^{2}}+\frac{1}{\beta_{n}^{2}}\left(u_{n, s}^{6}, u_{n}^{4}\right)_{L_{g}^{2}}-\frac{1}{\beta_{n}^{2}}\left(u_{n}^{4}, u_{n}^{4}\right)_{L_{g}^{2}} \longrightarrow 0 .
$$

Using (4.8) we have that $\frac{u_{n}^{4}}{\beta_{n}}$ is bounded in $H_{0}^{1}(0, L)$, and using (4.13) we get that the first term of (4.23) converges to zero. This yields

$$
b_{0}\left\|\frac{u_{n}^{4}}{\beta_{n}}\right\|_{H_{0}^{1}}^{2}-\frac{1}{\beta_{n}^{2}} \int_{0}^{\infty} g(s)\left(u_{n, s}^{6}, u_{n}^{4}\right)_{H_{0}^{1}} d s \quad \longrightarrow 0
$$

where $b_{0}:=\int_{0}^{\infty} g(s) d s$. We now prove that the second term in (4.24) converges to zero. In fact, using again that $\frac{u_{n}^{4}}{\beta_{n}}$ is bounded in $H_{0}^{1}(0, L),(3.12)$ and (4.12) we have

$$
\begin{aligned}
\left|-\frac{1}{\beta_{n}^{2}} \int_{0}^{\infty} g(s)\left(u_{n, s}^{6}, u_{n}^{4}\right)_{H_{0}^{1}} d s\right| & =\frac{1}{\left|\beta_{n}\right|}\left|-\int_{0}^{\infty} g^{\prime}(s)\left(u_{n}^{6}, \frac{u_{n}^{4}}{\beta_{n}}\right)_{H_{0}^{1}} d s\right| \\
& \leq \frac{k_{0}}{\left|\beta_{n}\right|}\left\|\frac{u_{n}^{4}}{\beta_{n}}\right\|_{H_{0}^{1}} \int_{0}^{\infty} g(s)\left\|u_{n}^{6}(s)\right\|_{H_{0}^{1}} d s \\
& \leq \frac{k_{0} \sqrt{b_{0}}}{\left|\beta_{n}\right|}\left\|\frac{u_{n}^{4}}{\beta_{n}}\right\|_{H_{0}^{1}}\left\|u_{n}^{6}\right\|_{L_{g}^{2}} \longrightarrow 0 .
\end{aligned}
$$

Therefore, we can deduce from (4.24) that

$$
\frac{u_{n}^{4}}{\beta_{n}} \longrightarrow 0 \quad \text { in } \quad H_{0}^{1}(0, L)
$$

it follows from (4.8) that

$$
u_{n}^{3} \quad \longrightarrow \quad 0 \quad \text { in } \quad H_{0}^{1}(0, L)
$$

and using (4.26) in (4.21) we get

$$
k\left\|u_{n, x}^{1}+u_{n}^{3}\right\|_{L^{2}}^{2} \longrightarrow \frac{1}{2}
$$

We want to show that this is a contradiction if the basic condition (4.1) holds.

Multiplying (4.9) by $\left(u_{n, x}^{1}+u_{n}^{3}\right)$ in $L^{2}(0, L)$ we have

$$
\begin{gathered}
i \beta_{n} \rho_{2}\left(u_{n}^{4}, u_{n, x}^{1}+u_{n}^{3}\right)_{L^{2}}+\left(\tilde{b} u_{n, x}^{3}+\int_{0}^{\infty} g(s) u_{n, x}^{6}(\cdot, s) d s,\left(u_{n, x}^{1}+u_{n}^{3}\right)_{x}\right)_{L^{2}} \\
+k\left\|u_{n, x}^{1}+u_{n}^{3}\right\|_{L^{2}}^{2}+\delta\left(u_{n, x}^{5}, u_{n, x}^{1}+u_{n}^{3}\right)_{L^{2}} \longrightarrow 0 .
\end{gathered}
$$

Note that by (4.13) we have that the last term of (4.28) converges to zero. Then we get

$$
\begin{aligned}
i \beta_{n} \rho_{2}\left(u_{n}^{4}, u_{n, x}^{1}+u_{n}^{3}\right)_{L^{2}}+\left(\tilde{b} u_{n, x}^{3}+\int_{0}^{\infty} g(s) u_{n, x}^{6}(\cdot, s) d s,\left(u_{n, x}^{1}+u_{n}^{3}\right)_{x}\right)_{L^{2}} & \\
+k\left\|u_{n, x}^{1}+u_{n}^{3}\right\|_{L^{2}}^{2} & \longrightarrow 0 .
\end{aligned}
$$


Also, multiplying (4.7) by $\frac{1}{k}\left(\tilde{b} u_{n, x}^{3}+\int_{0}^{\infty} g(s) u_{n, x}^{6}(\cdot, s) d s\right)$ in $L^{2}(0, L)$ results in

$$
-i \frac{\rho_{1}}{k} \beta_{n}\left(\tilde{b} u_{n, x}^{3}+\int_{0}^{\infty} g(s) u_{n, x}^{6}(\cdot, s) d s, u_{n}^{2}\right)_{L^{2}}-\left(\tilde{b} u_{n, x}^{3}+\int_{0}^{\infty} g(s) u_{n, x}^{6}(\cdot, s) d s,\left(u_{n, x}^{1}+u_{n}^{3}\right)_{x}\right)_{L^{2}}
$$

$\longrightarrow 0$.

Then, adding (4.29) and (4.30), we obtain

$$
i \beta_{n} \rho_{2}\left(u_{n}^{4}, u_{n, x}^{1}+u_{n}^{3}\right)_{L^{2}}-i \frac{\rho_{1}}{k} \beta_{n}\left(\tilde{b} u_{n, x}^{3}+\int_{0}^{\infty} g(s) u_{n, x}^{6}(\cdot, s) d s, u_{n}^{2}\right)_{L^{2}}+k\left\|u_{n, x}^{1}+u_{n}^{3}\right\|_{L^{2}}^{2}
$$

$\longrightarrow 0$.

On the other hand, multiplying (4.6) by $\rho_{2} u_{n}^{4},(4.8)$ by $i \rho_{2} \beta_{n} u_{n}^{3}$ and $(4.8)$ by $-\rho_{2} u_{n, x}^{2}$ in $L^{2}(0, L)$, respectively, yields

$$
\begin{aligned}
-i \beta_{n} \rho_{2}\left(u_{n}^{4}, u_{n, x}^{1}\right)_{L^{2}}-\rho_{2}\left(u_{n}^{4}, u_{n, x}^{2}\right)_{L^{2}} & \longrightarrow 0 \\
\beta_{n}^{2} \rho_{2}\left\|u_{n}^{3}\right\|_{L^{2}}^{2}+i \beta_{n} \rho_{2}\left(u_{n}^{4}, u_{n}^{3}\right)_{L^{2}} & \longrightarrow 0 \\
-i \beta_{n} \rho_{2}\left(u_{n}^{3}, u_{n, x}^{2}\right)_{L^{2}}+\rho_{2}\left(u_{n}^{4}, u_{n, x}^{2}\right)_{L^{2}} & \longrightarrow 0 .
\end{aligned}
$$

Since $u_{n}^{3} \rightarrow 0$ in $H_{0}^{1}(0, L) \hookrightarrow L^{2}(0, L)$, we obtain from (4.33)

$$
i \beta_{n} \rho_{2}\left(u_{n}^{4}, u_{n}^{3}\right)_{L^{2}} \longrightarrow 0 .
$$

Adding (4.31), (4.32), (4.34) and (4.35), we deduce that

$$
\begin{gathered}
-i \beta_{n} \rho_{2}\left(u_{n}^{3}, u_{n, x}^{2}\right)_{L^{2}}-i \frac{\rho_{1}}{k} \beta_{n}\left(\tilde{b} u_{n, x}^{3}+\int_{0}^{\infty} g(s) u_{n, x}^{6}(\cdot, s) d s, u_{n}^{2}\right)_{L^{2}}+k\left\|u_{n, x}^{1}+u_{n}^{3}\right\|_{L^{2}}^{2} \\
\longrightarrow 0 .
\end{gathered}
$$

Now, from (4.11) we have

$$
i \beta_{n} u_{n, x}^{6}+u_{n, s x}^{6}-u_{n, x}^{4} \quad \longrightarrow 0 \quad \text { in } \quad L_{g}^{2}\left(\mathbb{R}^{+}, L^{2}\right)
$$

then, multiplying by $\frac{\rho_{1}}{k} u_{n}^{2}$ in $L_{g}^{2}\left(\mathbb{R}^{+} ; L^{2}\right)$ results in

$$
i \beta_{n} \frac{\rho_{1}}{k}\left(u_{n, x}^{6}, u^{2}\right)_{L_{g}^{2}\left(\mathbb{R}^{+} ; L^{2}\right)}+\frac{\rho_{1}}{k}\left(u_{n, s x}^{6}, u^{2}\right)_{L_{g}^{2}\left(\mathbb{R}^{+} ; L^{2}\right)}-\frac{\rho_{1} b_{0}}{k}\left(u_{n, x}^{4}, u^{2}\right)_{L^{2}} \longrightarrow 0 .
$$

Using similar arguments used in (4.25) we can conclude

$$
\frac{\rho_{1}}{k}\left(u_{n, s x}^{6}, u^{2}\right)_{L_{g}^{2}\left(\mathbb{R}^{+} ; L^{2}\right)} \longrightarrow 0
$$

then it follows from (4.37) that

$$
\frac{\rho_{1} b_{0}}{k}\left(u_{n, x}^{4}, u^{2}\right)_{L^{2}} \longrightarrow 0
$$


Multiplying (4.8) by $-\frac{\rho_{1} b_{0}}{k} u_{n, x}^{2}$ in $L^{2}(0, L)$ yields

$$
i \beta_{n} \frac{\rho_{1} b_{0}}{k}\left(u^{3}, u_{n, x}^{2}\right)_{L^{2}}+\frac{\rho_{1} b_{0}}{k}\left(u_{n, x}^{4}, u^{2}\right)_{L^{2}} \longrightarrow 0
$$

then, adding (4.38) and (4.39), we get

$$
i \beta_{n} \frac{\rho_{1} b_{0}}{k}\left(u^{3}, u_{n, x}^{2}\right)_{L^{2}} \longrightarrow 0 .
$$

Finally, adding (4.36) and (4.40), we obtain

$$
-i \beta_{n} \rho_{2}\left(u_{n}^{3}, u_{n, x}^{2}\right)_{L^{2}}+i \frac{\rho_{1} \tilde{b}}{k} \beta_{n}\left(u_{n}^{3}, u_{n, x}^{2}\right)_{L^{2}}+i \frac{\rho_{1} b_{0}}{k} \beta_{n}\left(u_{n}^{3}, u_{n, x}^{2}\right)_{L^{2}}+k\left\|u_{n, x}^{1}+u_{n}^{3}\right\|_{L^{2}}^{2} \longrightarrow 0,
$$

and using that $\tilde{b}=b-b_{0}>0$, we obtain

$$
i \beta_{n} b(\underbrace{\frac{\rho_{1}}{k}-\frac{\rho_{2}}{b}}_{=0})\left(u_{n}^{3}, u_{n, x}^{2}\right)_{L^{2}}+k\left\|u_{n, x}^{1}+u_{n}^{3}\right\|_{L^{2}}^{2} \longrightarrow 0
$$

that is

$$
k\left\|u_{n, x}^{1}+u_{n}^{3}\right\|_{L^{2}}^{2} \longrightarrow 0
$$

which is contradiction to (4.27). Thus (4.2) is proved.

To complete the result about exponential stability we now prove (4.3). Note again that the resolvent equation $\left(i \lambda I d-\mathcal{A}_{3}\right) U=F \in \mathcal{H}_{3}$ is given by

$$
\begin{aligned}
i \lambda u^{1}-u^{2} & =f^{1} \\
i \lambda \rho_{1} u^{2}-k\left(u_{x}^{1}+u^{3}\right)_{x} & =\rho_{1} f^{2} \\
i \lambda u^{3}-u^{4} & =f^{3} \\
i \lambda \rho_{2} u^{4}-\tilde{b} u_{x x}^{3}-\int_{0}^{\infty} g(s) u_{x x}^{6}(\cdot, s) d s+k\left(u_{x}^{1}+u^{3}\right)+\delta u_{x}^{5} & =\rho_{2} f^{4} \\
i \lambda \rho_{3} u^{5}-\tilde{\beta} u_{x x}^{5}+\delta u_{x}^{4} & =\rho_{3} f^{5} \\
i \lambda u^{6}+u_{s}^{6}-u^{4} & =f^{6}
\end{aligned}
$$

where $b_{0}:=\int_{0}^{\infty} g(s) d s, \quad \tilde{b}:=b-b_{0}>0$. To prove (4.3) we will use a series of Lemmas.

Lemma 4.1 Let us suppose that the conditions (3.12) and (3.13) on g hold. Then there exists a positive constant $C$, being independent of $F$ such that

$$
\rho_{3} \int_{0}^{L}\left|u_{x}^{5}\right|^{2} d x+\iint_{0}^{L \infty} g(s)\left|u_{x}^{6}\right|^{2} d s d x \leq C\|U\|_{\mathcal{H}_{3}}\|F\|_{\mathcal{H}_{3}} .
$$


Proof. Multiplying $(4.42)$ by $u^{2}$ (in $\left.L^{2}(0, L)\right)$ we get

$$
i \lambda \rho_{2} \int_{0}^{L}\left|u^{2}\right|^{2} d x+k \int_{0}^{L}\left(u_{x}^{1}+u^{3}\right) \overline{u_{x}^{2}} d x=\rho_{1} \int_{0}^{L} f^{2} \overline{u^{2}} d x
$$

and, using equation (4.41),

$$
i \lambda \rho_{2} \int_{0}^{L}\left|u^{2}\right|^{2} d x-i \lambda k \int_{0}^{L}\left(u_{x}^{1}+u^{3}\right) \overline{u_{x}^{1}} d x=\rho_{1} \int_{0}^{L} f^{2} \overline{u^{2}} d x+k \int_{0}^{L}\left(u_{x}^{1}+u^{3}\right) \overline{f_{x}^{1}} d x .
$$

On the other hand, multiplying equation (4.44) by $u^{4}$ and integration over $[0, L]$ we get

$$
\begin{gathered}
i \lambda \rho_{2} \int_{0}^{L}\left|u^{4}\right|^{2} d x+\tilde{b} \int_{0}^{L} u_{x}^{3} \overline{u_{x}^{4}} d x+\underbrace{\int_{00}^{L \infty} g(s) u_{x}^{6} \overline{u_{x}^{4}} d s d x}_{=: I_{1}}+\underbrace{k \int_{0}^{L}\left(u_{x}^{1}+u^{3}\right) \overline{u^{4}} d x}_{=: I_{2}}+\delta \int_{0}^{L} u_{x}^{5} \overline{u_{4}} d x \\
=\rho_{2} \int_{0}^{L} f^{4} \overline{u^{4}} d x .
\end{gathered}
$$

Substituting $u^{4}$ given by (4.46), (4.43), into $I_{1}$ and $I_{2}$ we get

$$
\begin{gathered}
i \lambda \rho_{2} \int_{0}^{L}\left|u^{4}\right|^{2} d x-i \lambda \tilde{b} \int_{0}^{L}\left|u_{x}^{3}\right|^{2} d x-i \lambda \int_{0}^{L \infty} \int_{0}^{L} g(s)\left|u_{x}^{6}\right|^{2} d s d x-i \lambda k \int_{0}^{L}\left(u_{x}^{1}+u^{3}\right) \overline{u^{3}} d x \\
+\int_{0}^{L} \int_{0}^{L} g(s) u_{x}^{6} \overline{u_{x s}^{6}} d s+\delta \int_{0}^{L} u_{x}^{5} \overline{u^{4}} d x=\rho_{2} \int_{0}^{L} f^{4} \overline{u^{4}} d x+\tilde{b} \int_{0}^{L} u_{x}^{3} \overline{f_{x}^{3}} d x+k \int_{0}^{L}\left(u_{x}^{1}+u^{3}\right) \overline{f^{3}} d x \\
+\int_{0}^{L \infty} \int_{0}^{L} g(s) u_{x}^{6} \overline{f_{x}^{6}} d s d x
\end{gathered}
$$

Also, multiplying equation (4.45) by $\overline{u^{5}}$ we obtain

$$
i \lambda \rho_{3} \int_{0}^{L}\left|u^{5}\right|^{2} d x+\tilde{\beta} \int_{0}^{L}\left|u_{x}^{5}\right|^{2} d x-\delta \int_{0}^{L} u^{4} \overline{u_{x}^{5}} d x=\rho_{3} \int_{0}^{L} f^{5} \overline{u^{5}} d x .
$$

Adding (4.47), (4.48) and (4.49), using (3.12) and taking the real part our conclusion follows. Q.E.D.

Lemma 4.2 With the same hypotheses as in Lemma 4.1 there exists $C>0$ such that

$$
\rho_{2} \int_{0}^{L}\left|u^{4}\right|^{2} d x \leq C\|U\|_{\mathcal{H}_{3}}\|F\|_{\mathcal{H}_{3}}+C\|U\|_{\mathcal{H}_{3}}^{1 / 2}\|F\|_{\mathcal{H}}^{1 / 2}\left(\left\|u_{x}^{3}\right\|_{L^{2}}+\left\|u_{x}^{1}+u^{3}\right\|_{L^{2}}\right) .
$$


Proof. Multiplying (4.44) by $\int_{0}^{\infty} g(s) \overline{u^{6}} d s$ in $L^{2}(0, L)$ we get

$$
\begin{aligned}
& \underbrace{i \lambda \rho_{2} \iint_{00}^{L \infty} g(s) \overline{u^{6}} u^{4} d s d x}_{=: I_{3}}+\tilde{b} \iint_{0}^{L \infty} g(s) \overline{u_{x}^{6}} u_{x}^{3} d s d x+\int_{0}^{L}\left|\int_{0}^{\infty} g(s) u_{x}^{6} d s\right|^{2} d x \\
& \quad+k \iint_{0}^{L \infty} g(s)\left(u_{x}^{1}+u^{3}\right) \overline{u^{6}} d s d x-\delta \iint_{0}^{L \infty} g(s) \overline{u_{x}^{6}} u^{5} d s d x=\rho_{2} \iint_{0}^{L \infty} g(s) \overline{u^{6}} f^{4} d s d x .
\end{aligned}
$$

From Lemma 4.1 we obtain

$$
\int_{0}^{L}\left|\int_{0}^{\infty} g(s) u_{x}^{6} d s\right|^{2} d x \leq \int_{0}^{\infty} g(s) d s \int_{0}^{L \infty} \int_{0}^{\infty} g(s)\left|u_{x}^{6}\right|^{2} d s d x \leq C\|U\|_{\mathcal{H}}\|F\|_{\mathcal{H}_{3}}
$$

and

$$
\operatorname{Re}\left\{\delta \int_{0}^{L \infty} \int_{0}^{L \infty} g(s) \overline{u_{x}^{6}} u^{5} d s d x\right\} \leq C\|U\|_{\mathcal{H}}\|F\|_{\mathcal{H}_{3}} .
$$

Substituting $i \lambda u^{6}$ given by (4.45) into $I_{3}$, using

$$
\operatorname{Re}\left\{\iint_{0}^{L \infty} g(s) \overline{u_{s}^{6}} u^{4} d s d x\right\} \leq \frac{\rho_{2}}{2} \int_{0}^{L}\left|u^{4}\right|^{2} d x+C \int_{0}^{L} \int_{0}^{\infty}\left|g^{\prime}(s)\right|\left|u_{x}^{6}\right|^{2} d s d x
$$

and using (3.12), our conclusion now immediately follows from Lemma 4.1.

Q.E.D.

Lemma 4.3 With the same hypotheses as in Lemma 4.1, for any $\varepsilon_{1}>0$ there exists $C_{\varepsilon_{1}}>0$, at most depending on $\varepsilon_{1}$, such that

$$
\tilde{b} \int_{0}^{L}\left|u_{x}^{3}\right|^{2} d x \leq C_{\varepsilon_{1}}\|U\|_{\mathcal{H}}\|F\|_{\mathcal{H}_{3}}+C_{\varepsilon_{1}}\|U\|_{\mathcal{H}_{3}}^{1 / 2}\|F\|_{\mathcal{H}}^{1 / 2}\left\|u_{x}^{1}+u^{3}\right\|_{L^{2}}+\varepsilon_{1} \rho_{1}\left\|u^{2}\right\|_{L^{2}}^{2} .
$$

Proof. Multiplying of (4.44) by $u^{3}$ yields

$$
\begin{aligned}
& \underbrace{i \lambda \rho_{2} \int_{0}^{L} u^{4} \overline{u^{3}} d x}_{=: I_{4}}+\tilde{b} \int_{0}^{L}\left|u_{x}^{3}\right|^{2} d x+\int_{0}^{L} \int_{0}^{L} g(s) u_{x}^{6} \overline{u_{x}^{3}} d s d x \\
& \quad+k \int_{0}^{L}\left(u_{x}^{1}+u^{3}\right) \overline{u^{3}} d x+\delta \int_{0}^{L} u_{x}^{5} \overline{u^{3}} d x=\rho_{2} \int_{0}^{L} f^{4} \overline{u^{3}} d x .
\end{aligned}
$$


Substituting $i \lambda u^{3}$ given by (4.43) into $I_{4}$ we get

$$
\begin{aligned}
& \tilde{b} \int_{0}^{L}\left|u_{x}^{3}\right|^{2} d x+k \int_{0}^{L}\left(u_{x}^{1}+u^{3}\right) \overline{u^{3}} d x=\rho_{2} \int_{0}^{L}\left|u^{4}\right|^{2} d x \\
& \quad-\int_{0}^{L \infty} g(s) u_{x}^{6} \overline{u_{x}^{3}} d s d x+\delta \int_{0}^{L} u^{5} \overline{u_{x}^{3}} d x+\rho_{2} \int_{0}^{L} f^{4} \overline{u^{3}} d x+\rho_{2} \int_{0}^{L} u^{4} \overline{f^{3}} d x .
\end{aligned}
$$

On other hand, multiplying (4.42) by $\int_{0}^{x} \overline{u^{3}(y)} d y$ we get

$$
\underbrace{i \lambda \rho_{1} \int_{0}^{L} u^{2}\left(\int_{0}^{x} \overline{u^{3}(y)} d y\right) d x}_{=: I_{5}}-k \int_{0}^{L}\left(u_{x}^{1}+u^{3}\right)_{x}\left(\int_{0}^{x} \overline{u^{3}(y)} d y\right) d x=\rho_{1} \int_{0}^{L} f^{2}\left(\int_{0}^{x} \overline{u^{3}(y)} d y\right) d x
$$

Using (4.43) in $I_{5}$, we have

$$
I_{5}=-\rho_{1} \int_{0}^{L} u^{2}\left(\int_{0}^{x} \overline{u^{4}(y)} d y\right) d x-\rho_{1} \int_{0}^{L} u^{2}\left(\int_{0}^{x} \overline{f^{3}(y)} d y\right) d x .
$$

Substituting this into (4.51) we obtain

$$
\begin{aligned}
k \int_{0}^{L}\left(u_{x}^{1}+u^{3}\right) \overline{u^{3}} d x= & \rho_{1} \int_{0}^{L} u^{2}\left(\int_{0}^{x} \overline{u^{4}(y)} d y\right) d x+\rho_{1} \int_{0}^{L} u^{2}\left(\int_{0}^{x} \overline{f^{3}(y)} d y\right) d x \\
& +\rho_{1} \int_{0}^{L} f^{2}\left(\int_{0}^{x} \overline{u^{3}(y)} d y\right) d x .
\end{aligned}
$$

Finally, using (4.52) into (4.50) and using that

$$
\operatorname{Re}\left\{\rho_{1} \int_{0}^{L} u^{2} \overline{\int_{0}^{x} u^{4}(y) d y} d x\right\} \leq \varepsilon_{1} \rho_{1}\left\|u^{2}\right\|_{L^{2}}^{2}+C_{\varepsilon_{1}} \rho_{2}\left\|u^{4}\right\|_{L^{2}}^{2}
$$

taking real part (and using the Lemmas 4.2 and 4.1) our conclusion follows.

$$
\text { Q.E.D. }
$$

Our next step is to estimate the term $\left\|u_{x}^{1}+u^{3}\right\|_{L^{2}}^{2}$. Here we shall use condition (4.1).

Lemma 4.4 With the same hypotheses as in Lemma 4.1, together with condition (4.1), for any $\varepsilon_{2}>0$ there exists $C_{\varepsilon_{2}}>0$, at most depending on $\varepsilon_{2}$, such that

$$
k \int_{0}^{L}\left|u_{x}^{1}+u^{3}\right|^{2} d x \leq C_{\varepsilon_{2}}\|U\|_{\mathcal{H}_{3}}\|F\|_{\mathcal{H}_{3}}+\left(\varepsilon_{1}+\varepsilon_{2}\right) \rho_{1}\left\|u^{2}\right\|_{L^{2}}^{2}
$$

where $\varepsilon_{1}$ is given in Lemma 4.3. 
Proof. Multiplying (4.44) by $u_{x}^{1}+u^{3}$ we have

$$
\begin{aligned}
i \lambda \rho_{2} \int_{0}^{L} u^{4} \overline{\left(u_{x}^{1}+u^{3}\right)} & d x+k \int_{0}^{L}\left|u_{x}^{1}+u^{3}\right| d x+\delta \int_{0}^{L} u_{x}^{5} \overline{\left(u_{x}^{1}+u^{3}\right)} d x \\
& +\underbrace{\int_{0}^{L}\left[\tilde{b} u_{x}^{3}+\int_{0}^{\infty} g(s) u_{x}^{6} d s\right] \overline{\left(u_{x}^{1}+u^{3}\right)_{x}} d x}_{=: I_{6}}=\rho_{2} \int_{0}^{L} f^{4} \overline{\left(u_{x}^{1}+u^{3}\right)} d x .
\end{aligned}
$$

Substituting $\left(u_{x}^{1}+u^{3}\right)_{x}$ given by (4.42) into $I_{6}$ we get

$$
\begin{aligned}
& \underbrace{i \lambda \rho_{2} \int_{0}^{L} u^{4} \overline{u_{x}^{1}} d x}_{=: I_{7}}+\underbrace{i \lambda \rho_{2} \int_{0}^{L} u^{4} \overline{u^{3}} d x}_{=: I_{8}}-i \lambda \frac{\tilde{b} \rho_{1}}{k} \int_{0}^{L} u_{x}^{3} \overline{u^{2}} d x+k \int_{0}^{L}\left|u_{x}^{1}+u^{3}\right|^{2} d x \\
& +\delta \int_{0}^{L} u_{x}^{5} \overline{\left(u_{x}^{1}+u^{3}\right)} d x \underbrace{-i \lambda \frac{\rho_{1}}{k} \iint_{0}^{L \infty} g(s) u_{x}^{6} \overline{u^{2}} d s d x}_{=: I_{9}}-\frac{\rho_{1}}{k} \iint_{0}^{L \infty} g(s) u_{x}^{6} \overline{f^{2}} d s d x-\frac{\tilde{b} \rho_{1}}{k} \int_{0}^{L} u_{x}^{3} \overline{f^{2}} d x \\
& =\rho_{2} \int_{0}^{L} f^{4} \overline{\left(u_{x}^{1}+u^{3}\right)} d x .
\end{aligned}
$$

Substituting $u^{1}$ given by (4.41) and $u^{4}$ given by (4.43) into $I_{7}$ we obtain

$$
I_{7}=-i \lambda \rho_{2} \int_{0}^{L} u^{3} \overline{u_{x}^{2}} d x-\rho_{2} \int_{0}^{L} u^{4} \overline{f_{x}^{1}} d x+\rho_{2} \int_{0}^{L} f^{3} \overline{u_{x}^{2}} d x .
$$

Using (4.43) we get

$$
I_{8}=-\rho_{2} \int_{0}^{L}\left|u^{4}\right|^{2} d x-\rho_{2} \int_{0}^{L} u^{4} \overline{f^{3}} d x
$$

Finally, a substitution of $u^{6}$ given by (4.46) yields

$$
I_{9}=\frac{\rho_{1}}{k} \int_{0}^{L} \int_{0}^{\infty} g(s) u_{x s}^{6} \overline{u^{2}} d s d x-\frac{\rho_{1} b_{0}}{k} \int_{0}^{L} u_{x}^{4} \overline{u^{2}} d x-\frac{\rho_{1}}{k} \int_{0}^{L} \int_{0}^{\infty} g(s) f_{x}^{6} \overline{u^{2}} d s d x .
$$

From (4.43) we can rewrite $I_{9}$ as

$$
\begin{aligned}
I_{9}= & -\frac{\rho_{1}}{k} \int_{0}^{L} \int_{0}^{\infty} g^{\prime}(s) u_{x}^{6} \overline{u^{2}} d s d x-i \lambda \frac{\rho_{1} b_{0}}{k} \int_{0}^{L} u_{x}^{3} \overline{u^{2}} d x+\frac{\rho_{1} b_{0}}{k} \int_{0}^{L} f_{x}^{3} \overline{u^{2}} d x \\
& -\frac{\rho_{1}}{k} \int_{0}^{L} \int_{0}^{\infty} g(s) f_{x}^{6} \overline{u^{2}} d s d x .
\end{aligned}
$$


Using (4.54)-(4.56) in (4.53) we obtain

$$
\begin{gathered}
i \lambda b(\underbrace{\frac{\rho_{1}}{k}-\frac{\rho_{2}}{b}}_{=0}) \int_{0}^{L} u^{3} \overline{u_{x}^{2}} d x+k \int_{0}^{L}\left|u_{x}^{1}+u^{3}\right|^{2} d x=\rho_{2} \int_{0}^{L}\left|u^{4}\right|^{2} d x-\delta \int_{0}^{L} u_{x}^{5} \overline{\left(u_{x}^{1}+u^{3}\right)} d x \\
+\frac{\rho_{1}}{k} \int_{0}^{L} \int_{0}^{L} g^{\prime}(s) u_{x}^{6} \overline{u^{2}} d s d x+\frac{\rho_{1} \tilde{b}}{k} \int_{0}^{L} u_{x}^{3} \overline{f^{2}} d x+\frac{\rho_{1}}{k} \iint_{0}^{L \infty} g(s) u_{x}^{6} \overline{f^{2}} d s d x+\rho_{2} \int_{0}^{L} f^{4} \overline{\left(u_{x}^{1}+u^{3}\right)} d x \\
+\rho_{2} \int_{0}^{L} u^{4} \overline{f^{3}} d x+\rho_{2} \int_{0}^{L} u^{4} \overline{f_{x}^{1}} d x+\left(\rho_{2}-\frac{\rho_{1} b_{0}}{k}\right) \int_{0}^{L} f_{x}^{3} \overline{u^{2}} d x+\frac{\rho_{1}}{k} \int_{0}^{L \infty} \int_{0}^{L} g(s) f_{x}^{6} \overline{u^{2}} d s d x .
\end{gathered}
$$

Now, using (3.12) and the previous Lemmas, our claim follows.

Q.E.D.

Lemma 4.5 There exists $C>0$ such that

$$
\rho_{1} \int_{0}^{L}\left|u^{2}\right|^{2} d x \leq C\|U\|_{\mathcal{H}_{3}}\|F\|_{\mathcal{H}}+4 k\left\|u_{x}^{1}+u^{3}\right\|_{L^{2}}^{2} .
$$

Proof. Multiplying equation (4.42) by $u^{1}$ we get

$$
\underbrace{i \lambda \rho_{1} \int_{0}^{L} u^{2} \overline{u^{1}}}_{=: I_{10}} d x+k \int_{0}^{L}\left(u_{x}^{1}+u^{3}\right) \overline{u_{x}^{1}} d x=\rho_{1} \int_{0}^{L} f^{2} \overline{u^{1}} d x .
$$

Substitution of $u^{1}$ given by (4.41) into $I_{10}$ and taking real parts we get

$$
\rho_{1} \int_{0}^{L}\left|u^{2}\right|^{2} d x \leq C\|U\|_{\mathcal{H}_{3}}\|F\|_{\mathcal{H}}+2 k\left\|u_{x}^{1}+u^{3}\right\|_{L^{2}}^{2}+C\left\|u_{x}^{3}\right\|_{L^{2}}^{2} .
$$

Using Lemma 4.3 , for $\varepsilon_{1}$ sufficiently small, our conclusion follows.

Q.E.D.

Now we are in the position to prove the main result of this subsection.

Theorem 4.6 Let us assume hypothesis (3.12) and (3.13) on $g$ and suppose that condition (4.1) holds. Then the heat conducting Timoshenko system under the Fourier law is exponentially stable.

Proof. It remains to show (4.3).

Let $U=\left(u^{1}, u^{2}, u^{3}, u^{4}, u^{5}, u^{6}\right)^{\prime}, \quad F=\left(f^{1}, f^{2}, f^{3}, f^{4}, f^{5}, f^{6}\right)^{\prime}$ satisfy (4.41)-(4.46), then, from Lemma 4.1, we get

$$
\rho_{3}\left\|u^{5}\right\|_{L^{2}}^{2}+\left\|u^{6}\right\|_{L_{g}^{2}}^{2} \leq C\|F\|_{\mathcal{H}_{3}}\|U\|_{\mathcal{H}_{3}} .
$$


From Lemma 4.2 , for $\varepsilon_{2}>0$, there exists $C_{1}:=C_{1}\left(\varepsilon_{2}\right)>0$ such that

$$
\rho_{2}\left\|u^{4}\right\|_{L^{2}}^{2} \leq C_{1}\|F\|_{\mathcal{H}_{3}}\|U\|_{\mathcal{H}_{3}}+\frac{\tilde{b}}{2}\left\|u_{x}^{3}\right\|_{L^{2}}^{2}+\frac{\varepsilon_{2}}{2} k\left\|u_{x}^{1}+u^{3}\right\|_{L^{2}}^{2} .
$$

Also, from Lemma 4.3, we obtain

$$
\tilde{b}|| u_{x}^{3}\left\|_{L^{2}}^{2} \leq C_{\varepsilon_{1}}\right\| F\left\|_{\mathcal{H}_{3}}\right\| U\left\|_{\mathcal{H}_{3}}+\varepsilon_{1} \rho_{1}\right\| u^{2}\left\|_{L^{2}}^{2}+\frac{\varepsilon_{2}}{2} k\right\| u_{x}^{1}+u^{3} \|_{L^{2}}^{2} .
$$

Then, adding (4.58) and (4.59), we get

$$
\rho_{2}\left\|u^{4}\right\|_{L^{2}}^{2}+\frac{\tilde{b}}{2}|| u_{x}^{3}\left\|_{L^{2}}^{2} \leq C_{2}\right\| F\left\|_{\mathcal{H}_{3}}\right\| U\left\|_{\mathcal{H}_{3}}+\varepsilon_{1} \rho_{1}\right\| u^{2}\left\|_{L^{2}}^{2}+\varepsilon_{2} k\right\| u_{x}^{1}+u^{3} \|_{L^{2}}^{2} .
$$

On other hand, from Lemma 4.4 we have

$$
k\left\|u_{x}^{1}+u^{3}\right\|_{L^{2}}^{2} \leq C_{4}\|F\|_{\mathcal{H}_{3}}\|U\|_{\mathcal{H}_{3}}+\left(\varepsilon_{1}+\varepsilon_{2}\right) \rho_{1}\left\|u^{2}\right\|_{L^{2}}^{2} .
$$

Finally, from Lemma 4.5, we obtain

$$
2\left(\varepsilon_{1}+\varepsilon_{2}\right) \rho_{1}\left\|u^{2}\right\|_{L^{2}}^{2} \leq 2\left(\varepsilon_{1}+\varepsilon_{2}\right) C\|F\|_{\mathcal{H}_{3}}\|U\|_{\mathcal{H}_{3}}+8\left(\varepsilon_{1}+\varepsilon_{2}\right) k\left\|u_{x}^{1}+u^{3}\right\|_{L^{2}}^{2} .
$$

Adding (4.61) and (4.62) we conclude

$$
\left(1-8\left(\varepsilon_{1}+\varepsilon_{2}\right)\right) k\left\|u_{x}^{1}+u^{3}\right\|_{L^{2}}^{2}+\left(\varepsilon_{1}+\varepsilon_{2}\right) \rho_{1}\left\|u^{2}\right\|_{L^{2}}^{2} \leq C_{5}\|F\|_{\mathcal{H}_{3}}\|U\|_{\mathcal{H}_{3}} .
$$

From (4.57), (4.60) and (4.63), we obtain for $\varepsilon_{1}, \varepsilon_{2}$ sufficiently small, that there exists $C>0$ independent of $\lambda$ (and $F, U$ ) such that

$$
\|U\|_{\mathcal{H}_{3}}^{2} \leq C\|F\|_{\mathcal{H}_{3}}^{2}
$$

this completes the proof.

Q.E.D.

\subsection{Non-exponential stability for $\frac{\rho_{1}}{k} \neq \frac{\rho_{2}}{b}$}

Now we shall prove that condition (4.1) is also necessary for exponential stability.

Theorem 4.7 Let us suppose that (4.1) does not hold. Then the heat conduction Timoshenko system under the Fourier law is not exponentially stable.

Proof. As in Sections 2 and 3 it is sufficient to show that the solution of

$$
\left(i \lambda_{n} I d-\mathcal{A}_{3}\right) U_{n}=F_{n}
$$

satisfies

$$
\lim _{n \rightarrow \infty}\left\|U_{n}\right\|_{\mathcal{H}_{3}}=\infty
$$

where

$$
\lambda \equiv \lambda_{n}:=\frac{n \pi}{\alpha L} \quad(n \in \mathbb{N}), \quad \alpha:=\sqrt{\frac{\rho_{1}}{k}}
$$


and $F \equiv F_{n}:=(0, \cos (\alpha \lambda x), 0, \sin (\alpha \lambda x), 0,0)^{\prime}$. The solution $U_{n} \equiv U=\left(v^{1}, v^{2}, v^{3}, v^{4}, v^{5}, v^{6}\right)^{\prime}$ satisfies

$$
\begin{aligned}
i \lambda v^{1}-v^{2} & =0 \\
i \lambda v^{3}-v^{4} & =0 \\
-\lambda^{2} v^{1}-\frac{k}{\rho_{1}} v_{x x}^{1}-\frac{k}{\rho_{1}} v_{x}^{3} & =f^{2} \\
-\lambda^{2} v^{3}-\frac{b}{\rho_{2}} v_{x x}^{3}+\frac{b_{0}}{\rho_{2}} v_{x x}^{3}-\frac{1}{\rho_{2}} \int_{0}^{\infty} g(s) v_{x x}^{6}(x, s) d s+\frac{k}{\rho_{2}} v_{x}^{1}+\frac{k}{\rho_{2}} v^{3}+\frac{\delta}{\rho_{2}} v_{x}^{5} & =f^{4} \\
i \lambda v^{5}-\frac{\tilde{\beta}}{\rho_{3}} v_{x x}^{5}+i \lambda \frac{\delta}{\rho_{3}} v_{x}^{3} & =0 \\
i \lambda v^{6}+v_{s}^{6}-i \lambda v^{3} & =0 .
\end{aligned}
$$

This can be solved by

$$
\begin{gathered}
v^{1}(x)=A \cos (\alpha \lambda x), \quad v^{3}(x)=B \sin (\alpha \lambda x), \\
v^{5}(x)=C \cos (\alpha \lambda x), \quad v^{6}(x, s)=\varphi(s) \sin (\delta \lambda x)
\end{gathered}
$$

where $A, B, C, \varphi(s)$ depend on $\lambda$ and will be determined explicitly in the sequel. Note that this choice is again compatible with the boundary conditions. System (4.64) is equivalent to

$$
\begin{gathered}
-\lambda^{2} A+\frac{k}{\rho_{1}} \alpha^{2} \lambda^{2} A-\frac{k}{\rho_{1}} \alpha \lambda B=1 \\
-\lambda^{2} B+\frac{b}{\rho_{2}} \alpha^{2} \lambda^{2} B-\frac{b_{0}}{\rho_{2}} \alpha^{2} \lambda^{2} B+\frac{\alpha^{2} \lambda^{2}}{\rho_{2}} \int_{0}^{\infty} g(s) \varphi(s) d s-\frac{k}{\rho_{2}} \alpha \lambda A+\frac{k}{\rho_{2}} B-\frac{\delta \alpha}{\rho_{2}} \lambda C=1 \\
i \lambda C+\frac{\tilde{\beta}}{\rho_{3}} \alpha^{2} \lambda^{2} C+i \frac{\delta \alpha}{\rho_{3}} \lambda^{2} B=0 \\
i \lambda \varphi(s)+\varphi^{\prime}(s)-i \lambda B=0 .
\end{gathered}
$$

From (4.67) we have

$$
C=\frac{\lambda \delta \alpha}{i \tilde{\beta} \alpha^{2} \lambda-\rho_{3}} B
$$

On the other hand, by the definition of $\alpha$, we have we obtain from (4.65)

$$
B=-\frac{\rho_{1}}{k \alpha \lambda} .
$$

Since $v^{6}(0)=0$, we have that $\varphi(0)=0$, then solving (4.68) we get

$$
\varphi(s)=B-B e^{-i \lambda s} .
$$

From (4.71) we get

$$
\int_{0}^{\infty} g(s) \varphi(s) d s=\int_{0}^{\infty} g(s)\left[B-B e^{-i \lambda s}\right] d s=B b_{0}-B \int_{0}^{\infty} g(s) e^{-i \lambda s} d s .
$$


Let $\Theta:=\frac{b \rho_{1}}{\rho_{2} k}-1$. Then, using (4.69),(4.70) and (4.72) into (4.66) we have

$$
\frac{k \alpha \lambda}{\rho_{2}} A=-\Theta \lambda \alpha+\frac{\alpha^{3} \lambda}{\rho_{2}}\left(\int_{0}^{\infty} g(s) e^{-i \lambda s} d s\right)-\frac{k \alpha}{\rho_{2} \lambda}-1+\frac{\lambda \delta^{2} \alpha^{3}}{\left(i \alpha^{2} \lambda \tilde{\beta}-\rho_{3}\right) \rho_{2}}
$$

that is, using $\alpha=\sqrt{\frac{\rho_{1}}{k}}$,

$$
A=-\Theta \frac{\rho_{2}}{k}+\frac{\alpha^{2}}{k}\left(\int_{0}^{\infty} g(s) e^{-i \lambda s} d s\right)-\frac{\rho_{2}}{k \alpha \lambda}-\frac{1}{\lambda^{2}}+\frac{\delta^{2} \alpha^{2}}{\left(i \alpha^{2} \lambda \tilde{\beta}-\rho_{3}\right) k} .
$$

Recalling that $v^{2}=i \lambda v^{1}=i \lambda A \cos (\delta \lambda x)$, we get

$$
v^{2}(x)=\left(-i \lambda \frac{\rho_{2}}{k} \Theta+\frac{i \rho_{1}}{k^{2}} \lambda \int_{0}^{\infty} g(s) e^{-i \lambda s} d s-\frac{i \rho_{2}}{k \alpha}-\frac{i}{\lambda}+\frac{i \lambda \delta^{2} \alpha^{2}}{\left(i \alpha^{2} \lambda \tilde{\beta}-\rho_{3}\right) k}\right) \cos (\delta \lambda x) .
$$

Observing

$$
\begin{aligned}
\left\|v^{2}\right\|_{L^{2}} & =\left(\int_{0}^{L}\left|v^{2}\right|^{2} d x\right)^{1 / 2} \\
& =\frac{\sqrt{L}}{2}\left|-\lambda \frac{\rho_{2}}{k} \Theta+\frac{\rho_{1}}{k^{2}} \lambda \int_{0}^{\infty} g(s) e^{-i \lambda s} d s-\frac{\rho_{2}}{k \alpha}-\frac{1}{\lambda}+\frac{\lambda \delta^{2} \alpha^{2}}{\left(i \alpha^{2} \lambda \tilde{\beta}-\rho_{3}\right) k}\right| \\
& \geq \underbrace{-\frac{\sqrt{L}}{2}\left|-\frac{1}{\lambda}-\frac{\rho_{2}}{\sqrt{\rho_{1} k}}+\frac{\rho_{1}}{k^{2}} \lambda \int_{0}^{\infty} g(s) e^{-i \lambda s} d s+\frac{\lambda \delta^{2} \alpha^{2}}{\left(i \alpha^{2} \lambda \tilde{\beta}-\rho_{3}\right) k}\right|}_{\text {bounded as } \lambda \rightarrow \infty}+\frac{\sqrt{L}}{2}|\underbrace{\frac{\rho_{2}}{b}-\frac{\rho_{1}}{k}}_{\neq 0}| \frac{b}{k} \lambda
\end{aligned}
$$

and using Lemma 3.2, we obtain

$$
\lim _{\lambda \rightarrow \infty}\left\|U_{n}\right\|_{\mathcal{H}_{3}} \geq \lim _{\lambda \rightarrow \infty}\left\|v^{2}\right\|_{L^{2}}=\infty
$$

which completes the proof.

Q.E.D.

\section{References}

[1] Ammar Khodja, F., Benabdallah, A., Muñoz Rivera, J.E., Racke R.: Energy decay for Timoshenko systems of memory type. J. Differential Equations 194 (2003), 82-115.

[2] Chandrasekharaiah, DS.: Hyperbolic thermoelasticity: a review of recent literature. Appl. Mech. Rev. 51 (1998), 705-729.

[3] Fernández Sare, H.D., Muñoz Rivera, J.E.: Stability of Timoshenko systems with past history. Submitted (2006). 
[4] Grasselli, M., Muñoz Rivera, J.E., Pata, V.: On the energy decay of the linear thermoelastic plate with memory. J. Math. Anal. Appl. 309 (2005), 1-14.

[5] Irmscher, T.: Aspekte hyperbolischer Thermoelastizität. Thesis, University of Konstanz (2006) (english version in preparation).

[6] Irmscher, T., Racke, R.: Sharp decay rates in parabolic and hyperbolic thermoelasticity. IMA J. APpl. Math. 71 (2006), 459-478.

[7] Jiang, S. and Racke, R.: Evolution equations in thermoelasticity. $\pi$ Monographs Surveys Pure Appl. Math. 112, Chapman \& Hall/CRC, Boca Raton (2000).

[8] Liu, Z.: Energy decay rate of the thermoelastic Bresse system. Preprint (2004).

[9] Liu, Z., Zheng, S.: Semigroups associated with dissipative systems. $\pi$ Research Notes Math. 398, Chapman\&Hall/CRC, Boca Raton (1999).

[10] Messaoudi, S.A., Said-Houari, B.: Exponential stability in one-dimensional non-linear thermoelasticity with second sound. Math. Meth. Appl. Sci. 28 (2005), 205-232.

[11] Muñoz Rivera, J.E., Racke, R.: Mildly dissipative nonlinear Timoshenko systems — global existence and exponential stability. J. Math. Anal. Appl. 276 (2002), 248-278.

[12] Muñoz Rivera, J.E., Racke, R.: Global stability for damped Timoshenko systems. Disc. Cont. Dyn. Sys. 9 (2003), 1625-1639.

[13] Öncü, S.T., Moodie, B.T.: On the constitutive relations for second sound in elastic solids. Arch. Rational Mech. Anal. 121 (1992), 87-99.

[14] Racke, R.: Thermoelasticity with second sound - exponential stability in linear and nonlinear 1-d. Math. Meth. Appl. Sci. 25 (2002), 409-441.

[15] Racke, R.: Asymptotic behavior of solutions in linear 2- or 3-d thermoelasticity with second sound. Quart. Appl. Math. 61 (2003), 315-328.

[16] Soufyane, A.: Stabilisation de la poutre de Timoshenko. C. R. Acad. Sci. Paris, Sér. I 328 (1999), 731-734.

Hugo D. Fernández Sare, Department of Mathematics and Statistics, University of Konstanz, 78457 Konstanz, Germany

hugo.fernandez-sare@uni-konstanz.de

Reinhard RACKE, Department of Mathematics and Statistics, University of Konstanz, 78457 Konstanz, Germany

reinhard.racke@uni-konstanz.de 